Part of Journal of Research of the National Bureau of Standards, Volume 32, March 1944

\title{
THERMAL EXPANSION OF CONCRETE AGGREGATE MATERIALS
}

\author{
By Walter H. Johnson and Willard H. Parsons
}

\section{ABSTRACT}

As a part of a study of the properties of concrete aggregates, thermal-expansion determinations were made on 123 specimens of aggregate materials by the optical interferometer method over the temperature range $-20^{\circ}$ to $+60^{\circ} \mathrm{C}$. Additional measurements were made on crystals of calcite, quartz, and feldspar: The thermal expansivities of most aggregate materials are close to or within the range of expansivities of hardened portland cements. Certain exceptions are pointed out. Crystal orientation, rock texture, and composition are discussed with regard to their effects on the relation of the thermal expansion of aggregates to the durability of concrete.

\section{CONTENTS}

I. Introduction

II. Previous work

III. Test method

1. Apparatus and calibration

(a) General description

(b) Establishment of heating and cooling schedule _..... 104

(c) Calibration of thermocouple and refraction thermometer

(d) Determination of temperature lag in test specimen... 105

2. Preparation of specimens.

3. Test procedure

4. Reliability of results

IV. Description of materials tested

V. Results and discussion

1. Effect of crystal orientation

2. Effect of composition difference

3. Effect of texture

4. Materials with irregular expansion $\ldots \ldots \ldots \ldots 121$

5. Comparison of present results with previous work

6I. Aggregate expansivity in relation to concrete

VII References_...

\section{INTRODUCTION}

In concrete structures, where resistance to frost action is one of the properties required of the concrete, the thermal expansivity of the aggregate may be an important factor. If, for instance, the thermal expansion of an aggregate differs considerably from that of the cementitious matrix, a potential incompatability exists, which may result in excessive internal stresses if the concrete is subjected to large temperature changes. Pearson $\lceil 1\rceil^{1}$ and also Hornibrook [2]

1 Figures in brackets indicate the literature references at the end of this paper. 
have described concrete failures attributed to an initial weakening of the concrete because of differences between the thermal expansion of the aggregates (which had unusually low coefficients of thermal expansion) and that of the matrix. The weakened concrete then became susceptible to frost action, which caused the actual disruption. This sequence of disintegration has recently been demonstrated in the laboratory by Pearson [3].

Adequate information is lacking on the thermal expansion characteristics of commercial concrete aggregates. Accordingly, as a part of a general study at this Bureau of the properties of aggregate materials, commercial aggregates from a number of sources were obtained and separated into fractions representing the various rocks and minerals constituting the aggregates. Coefficients of linear thermal expansion were then determined for the rocks and minerals over the temperature range of $-20^{\circ}$ to $+60^{\circ} \mathrm{C}\left(-4^{\circ}\right.$ to $\left.+140^{\circ} \mathrm{F}\right)$. This paper gives a petrographic description of the various rock and mineral fractions of the aggregates, the respective thermal-expansion coefficients of these materials, and a discussion of the factors which may effect the thermal expansion in relation to concrete.

Some problems encountered during the present investigation warrant further study, and additional thermal-expansion measurements should be made on certain of the aggregate materials herein considered. Due to the pressure of the wartime emergency, however, these studies cannot be continued at this time.

\section{PREVIOUS WORK}

Griffith [4] has made thermal-expansion measurements on a considerable number of rocks from room temperature to $500^{\circ} \mathrm{F}$; he also made some measurements between $32^{\circ} \mathrm{F}$ and room temperature. Willis and De Reus [5] measured the thermal expansion of wet and dry specimens of chert, quartzite, sandstone, basalt, granite, limestone, and dolomite between $37^{\circ}$ and $140^{\circ} \mathrm{F}$. Koenitzer [6] measured the expansion of marble and calcite between $-25^{\circ}$ and $+212^{\circ} \mathrm{F}$. Souder and Hidnert [7] have made thermal-expansion measurements on marble and have shown that permanent changes occur with succeeding temperature cycles. (A comparison of some of these results with those of the present investigation will be given later.) Davis [8] and others [5, 6, and 9] have measured the thermal expansion of hardened portland cements, mortars, and concretes under various conditions. They have shown the effects of such factors as water saturation, extreme drying, steaming, richness of mix, and age on the thermal expansion.

An examination of the available data shows that there is very little information regarding the thermal dilation of the aggregate materials within the temperature range of natural weather conditions. The majority of investigators, furthermore, have assumed a more or less straight-line relationship between temperature and thermal expansion, without regard to possible irregularities during the large intervals between readings. Although the expansion of various materials, when plotted against temperature, usually yield smooth curves, it will be shown that there are some glaring exceptions. In this investigation, therefore, the length changes of the aggregate materials have been followed continuously from $-25^{\circ}$ to $+65^{\circ} \mathrm{C}$ and from $+65^{\circ}$ to $-5^{\circ} \mathrm{C}$. 


\section{TEST METHOD}

\section{APPARATUS AND CALIBRATION}

(a) GENERAL DESCRIPTION

The interferometer method described by Merritt [10] and modified by Saunders [11] was used in conjunction with a thermal chamber that could be varied continuously between $-25^{\circ}$ and $+65^{\circ} \mathrm{C}$. This permitted the use of small specimens, the measurement of low expansivities, and the taking of frequent observations separated by small temperature intervals. The thermal chamber (fig. 1) consisted essentially of a closed brass cylinder, about 9 in. high and 3 in. in diameter, inserted through the top of a remodeled household refrigerator into a tank of kerosine which surrounded the freezing coils. A hard-rubber tube wound with $30 \mathrm{ft}$. of No. 24 Nichrome resistance wire served as

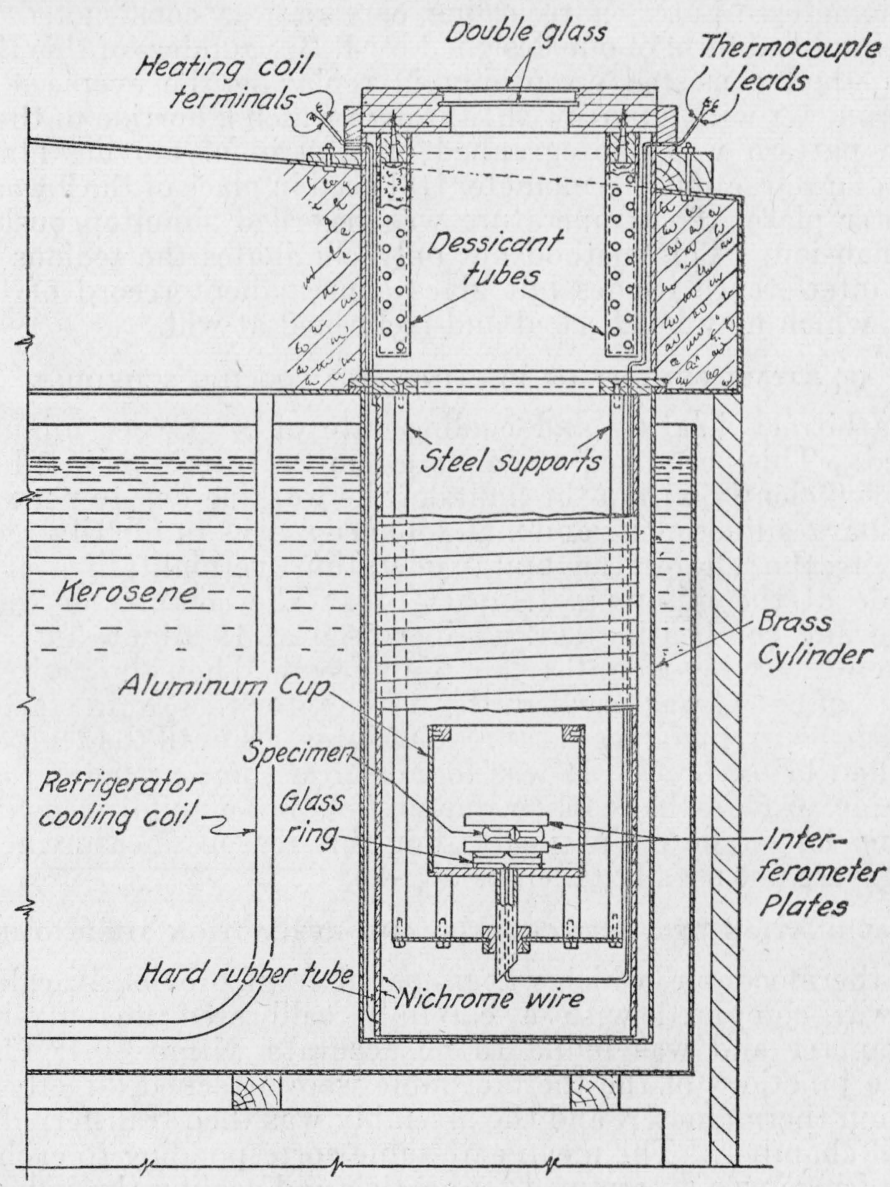

Vertical section

FiguRE 1.-Schemalic diagram of thermal chamber. 


\section{Journal of Research of the National Bureau of Standards}

the heating element. The specimen and interferometer plates were supported from the top of the cylinder by $1 / 4$-in. steel rods. The top was closed by means of a plastic lid fitted with a double glass window. Perforated brass tubes were filled with absorbent cotton, which served as a desiccant. These tubes were removable and were dried at $105^{\circ}$ to $110^{\circ} \mathrm{C}$ before each test. The lower interferometer plate was separated from the bottom of the aluminum specimen cup by means of a glass ring cut from a piece of tubing and ground in such a way that there were three equidistant bearing surfaces on the top and three on the bottom.

About half of the expansion measurements were made by the visual method. The temperatures were measured by means of a threejunction copper-constantan thermocouple [12], the variable junctions of which were situated just beneath the lower interferometer plate. The emf of the thermocouple was measured by means of a portable potentiometer. Later, a recording camera was constructed, which was a simplified form of one designed by J. B. Saunders of this Bureau. This method consisted essentially in replacing the eyepiece of the interferometer with a narrow slit through which a portion of the interference pattern was photographed on a strip of moving film. By means of a refraction thermometer [13] used in place of the lower interferometer plate, the temperature was recorded simultaneously with the expansion. This method not only eliminates the tedious counting of interference fringes but gives a permanent record of the behavior, which may be checked and rechecked at will.

\section{(b) ESTABLISHMENT OF HEATING AND COOLING SCHEDULE}

An arbitrary heating and cooling rate of $1 / 20 \mathrm{C}$ per minute was adopted. This permitted a complete temperature cycle in 6 hours, a period sufficiently within the limits of the working day to permit such preliminary adjustments and maintenance as was ordinarily required. By leaving the refrigerating unit in operation overnight, the start could be made at the minimum temperature. The compressor was then stopped and the heating current increased at 15-minute intervals in increments varying from 0.1 to 0.2 ampere. When the desired temperature of $65^{\circ} \mathrm{C}$ was reached, the refrigerator was again started and the heating current decreased at regular intervals until the temperature had fallen below $0^{\circ} \mathrm{C}$. It was found, after some preliminary trials, that below $0^{\circ} \mathrm{C}$ further cooling was so slow that a uniform cooling rate could no longer be maintained. For this reason, measurements on cooling were not usually taken below $-5^{\circ} \mathrm{C}$.

\section{(c) CALIBRATION OF THERMOCOUPLE AND REFRACTION THERMOMETER}

The thermocouple, which was prepared from previously calibrated wire, was compared with a carefully calibrated mercury-in-glass thermometer and was found to be accurate within $\pm 0.1^{\circ} \mathrm{C}$. The variable junctions of the thermocouple were attached directly to the refraction thermometer, and the assembly was then transferred to the thermal chamber. The mean emf value corresponding to each interference fringe was determined by heating and cooling through several temperature cycles, according to schedule, and recording the emf for the passage of each fringe across the reference mark of the thermometer. The accuracy of the temperatures, as measured by this refraction thermometer, was found to be within $\pm 0.2^{\circ} \mathrm{C}$. 
(d) DETERMINATION OF TEMPERATURE LAG IN TEST SPECIMEN

The actual temperature of the specimen, for a given thermocouple reading, was determined by means of a small quartz refraction thermometer placed between the interferometer plates and separated from them by small grains of sand. The temperature reading of the thermometer was found to lag several degrees behind that of the thermocouple when heating or cooling. As this thermometer had about the same mass as that of the specimens to be measured, the assumption was made that the temperature lag would be about the same in each case. Several temperature cycles were made according to the current-time schedule previously adopted, and emf-temperature curves were drawn from the data obtained. These curves were used to determine the temperatures under actual operating conditions. When measurements were made with the recording apparatus the temperature of the specimen was assumed to be the same as that of the refraction thermometer.

\section{PREPARATION OF SPECIMENS}

The majority of the specimens were taken from gravel ranging from $3 / 4$ in to $1 \frac{1 / 2}{2}$. in diameter. A few were taken from larger pieces of stone, and in some cases specimens were cut from material less than $1 / 2$ in. in diameter. Figure 2 (a, b, c, d, e, and f) shows, schematically, the successive steps in the preparation of a test specimen. Slabs (a), ranging in thickness from 0.2 in. to 0.4 in., were cut from each specimen, and triangular pieces (b), having sides of about $3 / 4$ in., were cut

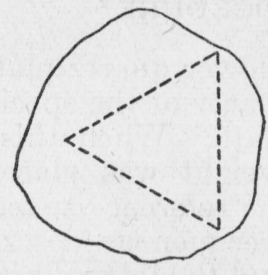

(a)

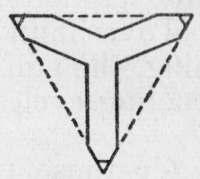

(c)

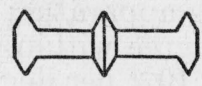

(e)

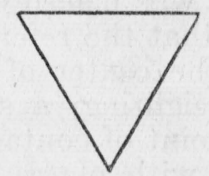

(b)

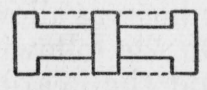

(d)

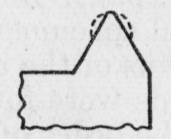

(f)

Figure 2.-Schematic diagrams to show successive steps in the preparation of a test specimen.

(a) Original flat slab marked for further cutting; (b) triangular slab; (c) arms cut from triangular slab leaving a Y-shaped specimen; (d) side view of (c) after grinding it to form the spacers; (e) points cut on spacers; $(f)$ enlarged part of one spacer, showing rounding of its point. 


\section{Journal of Research of the National Bureau of Standards}

from the slabs. The sides of the triangular-shaped pieces were prepared by grinding on a small silicon carbide wheel to leave a $Y$-shaped specimen (c) having one arm slightly longer than the others. Portions of the top and bottom of each arm were then ground to form two small projections (d), these two projections forming a spacer. The spacers were pointed as shown (e), care being taken that the top point of each spacer was vertically above lower contact point. By means of a micrometer gage and an emery stone, the heights of the spacers were equalized to within $0.001 \mathrm{in}$. The projections were slightly rounded (f) by rubbing on a very fine emery stone. The spacers of the finished specimens were about 0.25 in. in height.

The nearly completed specimen was placed between interferometer plates and examined through a viewing instrument. The interference pattern observed usually consisted of very narrow fringes, and a slight pressure on the upper plate caused these fringes to move toward the tallest spacer of the specimen [10]. The heights of the spacers were adjusted by grinding them carefully until the fringes were of the desired width and the tallest spacer was on the longest arm [11]. The tips of the spacers were usually polished sufficiently in the process to show the points of contact with the upper interferometer plate as tiny spots surrounded by Newton rings, and the point on the longest arm was used as a reference point for counting the fringes. When this point could not be seen, a small grain of sand or a piece of fine wire was placed directly over the estimated point of contact for reference. The finished specimen was placed in a desiccator containing calcium chloride for several days before testing.

\section{TEST PROCEDURE}

The specimen was placed on the lower quartz plate and the upper plate placed so that the reference spacer of the specimen was as near as possible to the center of the plate. When this did not permit sufficient overweighting, a small weight was placed on the upper plate near the point of contact of the reference spacer with the plate [11]. The cup, with plates and specimen as described above, was lowered into the thermal chamber, and the lid and viewing instrument were placed in position. The temperature was then raised to about $65^{\circ} \mathrm{C}$ to remove as nearly as possible any air film between the points of contact of the specimen with the interferometer plates, after which the thermal chamber was allowed to cool overnight with the refrigerator in operation. On the following morning, the emf and fringe readings were recorded and the heating and cooling cycles carried through as previously described.

The measurements were repeated for those materials which displayed abnormal expansivities or whenever an error in the data was suspected. Several specimens, measured by the visual method, were remeasured by means of the recording apparatus; the results obtained for the two methods were found to agree within the limits of experimental error (on the order of $\pm 0.1 \times 10^{-6}$ per degree centigrade). 


\section{RELIABILITY OF RESULTS}

In order to test the performance of the apparatus and the accuracy of the calibrations, samples of known thermal expansion were obtained from the Thermal Expansion Section of this Bureau and were tested in the manner described. A comparison of the results obtained is as follows:

\begin{tabular}{|c|c|c|c|}
\hline \multirow{2}{*}{ Sample } & \multirow{2}{*}{$\begin{array}{c}\text { Temperature } \\
\text { interval }\end{array}$} & \multicolumn{2}{|c|}{$\begin{array}{l}\text { Coefficient of linear thermal } \\
\text { expansion per degree centigrade }\end{array}$} \\
\hline & & Hidnert a & Johnson \\
\hline $\begin{array}{l}P \\
Q \\
Q \\
Q \\
Q \\
R\end{array}$ & $\begin{array}{r}{ }^{\circ} C \\
20 \text { to } 60 \\
-20 \text { to } 0 \\
0 \text { to } 20 \\
20 \text { to } 60 \\
20 \text { to } 60\end{array}$ & $\begin{array}{l}\text { 29. } 0 \times 10^{-6} \\
\text { 11. } 0 \times 10^{-6} \\
\text { 11. } 3 \times 10^{-6} \\
\text { 12. } 1 \times 10^{-6} \\
0.38 \times 10^{-6}\end{array}$ & $\begin{array}{r}29.1 \times 10^{-6} \\
\text { 11. } 0 \times 10^{-6} \\
11.3 \times 10^{-6} \\
12.1 \times 10^{-6} \\
0.4 \times 10^{-6}\end{array}$ \\
\hline
\end{tabular}

a Peter Hidnert, Thermal Expansion Section, National Bureau of Standards

\section{DESCRIPTION OF MATERIALS TESTED}

Commercial aggregates from 26 different sources were divided into various rock and mineral fractions (table 1). Specimens of these rocks and minerals and of some related materials, whose thermal expansion behaviors are reported in the present study, were examined with a petrographic microscope. The results of these examinations, together with designations of source, classification, and texture, are shown in table 2. The mineral compositions were determined by microscopic study of thin sections and the relative amounts or percentages of each mineral were estimated without a quantitative microscopic count. The percentage values given in table 2 are, therefore, approximations. The naming, or classification, of the rocks has followed common geologic and petrographic usage.

Under the heading of texture (table 2), noncrystalline materials are described as amorphous or glassy; specimens cut from single crystals are so indicated; and the grain size is given for minerals or rocks composed of many particles or crystals. The grain-size terminology refers to actual sizes in millimeters as follows:

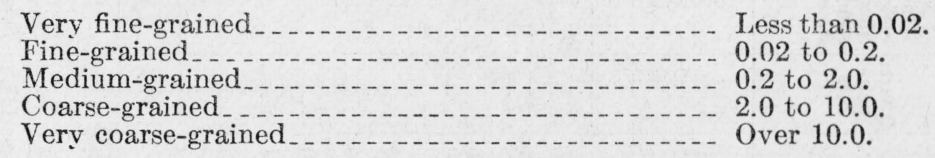

For rocks of porphyritic texture (most of the rocks between specimens 75 and 94), the grain size in the groundmass is given first and is followed by the grain size of the larger crystals, or so-called phenocrysts. Similarly, with breccias (some of the rocks between specimens 95 and 104), the grain sizes of both the groundmass and of the larger fragments are given. 
TABLE 1.-Approximate percentages of rock and mineral fractions in various commercial concrete aggregates

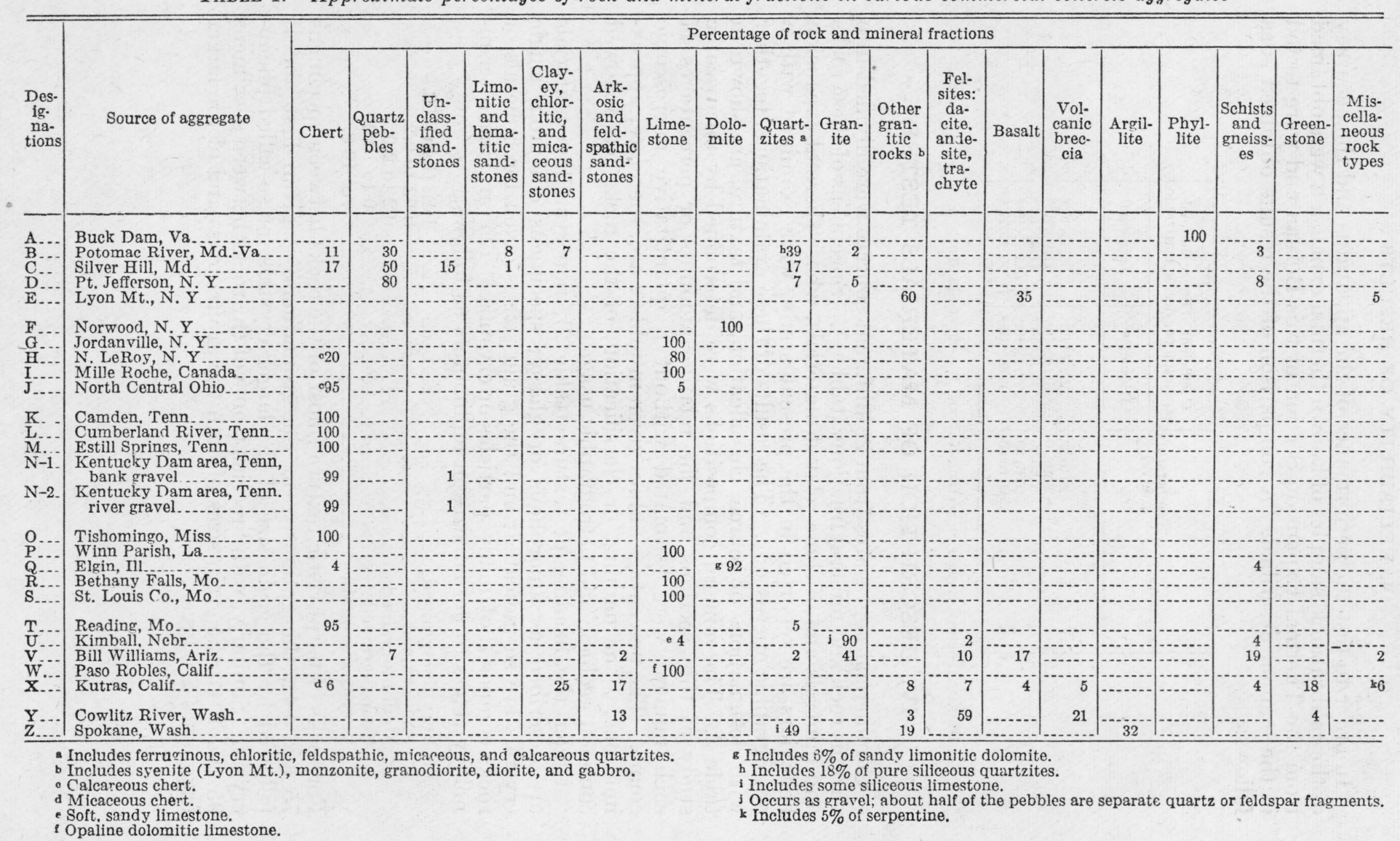


TABLE 2.-Description, source, thermal-expansion coefficients, texture, and mineral composition of all materials tested

\begin{tabular}{|c|c|c|c|c|c|c|c|c|c|}
\hline \multirow{2}{*}{$\begin{array}{c}\text { Speci- } \\
\text { men } \\
\text { num- } \\
\text { ber }\end{array}$} & \multirow[t]{2}{*}{ Description } & \multirow[t]{2}{*}{ Source } & \multirow{2}{*}{$\begin{array}{c}\text { Des- } \\
\text { igna- } \\
\text { tion } \\
\text { in } \\
\text { table } \\
1\end{array}$} & \multicolumn{3}{|c|}{$\begin{array}{l}\text { Mean coefficient of } \\
\text { linear thermal ex- } \\
\text { pansion per degree } \\
\mathrm{C} \times 10^{6}\end{array}$} & \multirow[t]{2}{*}{ Texture } & \multicolumn{2}{|c|}{$\begin{array}{c}\text { Mineral constituents b (in decreasing order of abundance; } \\
\text { percentages approximate only) }\end{array}$} \\
\hline & & & & $\begin{array}{l}-20^{\circ} \text { to } \\
+60^{\circ}\end{array}$ & $\begin{array}{l}0^{\circ} \text { to } \\
+60^{\circ}\end{array}$ & $\begin{array}{l}+60^{\circ} \\
\text { to } 0^{\circ}\end{array}$ & & Major, more than 3 percent & Minor, less than 3 percent \\
\hline 1 & Quartz (measured perpen- & Minas Geraes, Brazil & & 13.5 & 14.0 & 13.5 & Single crysta & Quartz & \multirow{11}{*}{$\begin{array}{l}\text { Trace of limonite. } \\
\text { Do. }\end{array}$} \\
\hline 2 & $\begin{array}{l}\text { dicular to } c \text {-axis) } \\
\text { Quartz (measured parallel }\end{array}$ & - do & & 7.4 & 7.7 & 7.7 & do & Same crystal as specimen 1. & \\
\hline 3 & $\begin{array}{l}\text { to c-axis) } \\
\text { Quartz, pale yellow .. }\end{array}$ & Potomac R & B & 12.9 & 13.1 & 13.5 & Coarse and fine. & Quartz_.. & \\
\hline 4 & .......do & Va. & B & 12.7 & 12.8 & 13.5 & ..... do. & ..... do & \\
\hline 5 & Quartz, milky & $\begin{array}{l}\text { Port Jefferson gravel, Long } \\
\text { Island, N. Y. }\end{array}$ & $\mathrm{D}$ & 13.5 & 13.8 & 13.8 & Coarse. & & \\
\hline 6 & Quartz, pale yellow & - do & $\mathrm{D}$ & 11.8 & 11.8 & 12.0 & ......do & 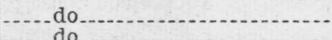 & \\
\hline $\begin{array}{l}7 \\
8\end{array}$ & $\begin{array}{l}\text { Quartz, colorless } \\
\text { Quartzite, light-colored }\end{array}$ & Potomac River gravel, & $\underset{B}{D}$ & $\begin{array}{l}11.7 \\
11.3\end{array}$ & $\begin{array}{l}12.8 \\
12.0\end{array}$ & $\begin{array}{l}12.4 \\
12.9\end{array}$ & Medium & Quartz $99 \%$ & \\
\hline 9 & & $\begin{array}{l}\text { Md.-Va. } \\
\text { do }\end{array}$ & B & & 10.4 & 10.9 & & & \\
\hline 10 & Quart & ..... do .. & B & 11.9 & 12.1 & 12.7 & & $95 \%$, goethite $4 \%$ & \\
\hline 11 & $\begin{array}{l}\text { Quart } \\
\text { Quart }\end{array}$ & do do & B & $\begin{array}{r}10.7 \\
7.0\end{array}$ & 11.1 & $\begin{array}{r}11.1 \\
7.5\end{array}$ & & $94 \%$, chlorite $5 \%$ & \\
\hline 12 & Ielaspatm & $\begin{array}{l}\text { Port Jefferson gravel, Long } \\
\text { Island, N. Y. }\end{array}$ & D & & & & Me & Quar & \\
\hline $\begin{array}{l}13 \\
14\end{array}$ & Quartzite, light-colored & spokane gravel, Irvin, Wash & $\begin{array}{l}\mathrm{D} \\
\mathrm{Z}\end{array}$ & $\begin{array}{r}12.2 \\
7.6\end{array}$ & $\begin{array}{r}12.5 \\
7.9\end{array}$ & $\begin{array}{r}12.8 \\
8.4\end{array}$ & Fine.............. & $\begin{array}{l}\text { Quartz, feldspar, biotite....... } \\
\text { Quartz } 93 \% \text {, sericite } 3 \%\end{array}$ & \multirow{2}{*}{$\begin{array}{l}\text { Musc. } 2 \% \text {, calcite } 2 \% \text {, plagio- } \\
\text { clase. } \\
\text { Trace of titanite, leucoxene. }\end{array}$} \\
\hline 15 & Quartzite, micaccous... & .....do & $\mathrm{z}$ & 9.0 & 8.7 & 9.8 & ... do & , sericite $25 \%$, bio- & \\
\hline 16 & $\begin{array}{l}\text { Quartzite, calcareous mica- } \\
\text { ceous. }\end{array}$ & \multirow{3}{*}{$\begin{array}{l}\text { Potomac River gravel, Md.- } \\
\text { Va. }\end{array}$} & $z$ & 9.5 & 9.3 & 9.8 & ...... do. & \multirow{6}{*}{$\begin{array}{l}\text { tite } 10 \% \text {. } \\
\text { Quartz } 55 \% \text {, cal. } 25 \% \text {, ground- } \\
\text { mass of microq. and sericite } \\
20 \% \text {. } \\
\text { Quartz } 94 \% \text {, hematite } 3 \% \text {-..... } \\
\text { Quartz } 94 \% \text {, limo. and goe- } \\
\text { thite } 5 \% \text {. } \\
\text { Quartz } 96 \% \text {, limo. and goe- } \\
\text { thite } 3 \% . \\
\text { Quartz } 92 \% \text {, limo. and goe- } \\
\text { thite (clayey and carb.) } 8 \% \text {. } \\
\text { Quartz } 80 \% \text {, sericite } 10 \% \text {, chlo- } \\
\text { rite } 6 \% \text {. }\end{array}$} & \multirow{6}{*}{$\begin{array}{l}\text { Musc. } 2 \% \text {, pc.; traces of titan- } \\
\text { ite and leucoxene. } \\
\text { Mica } 3 \% \text {; traces pc., limo., py. } \\
\text { Chalc.; traces of mica, feldspar } \\
\text { titanite. } \\
\text { Chalc.; traces of mica, feld- } \\
\text { spar, zircon. } \\
\text { Mica; traces chalc., feldspar, } \\
\text { titanite, zircon. } \\
\text { Limo. } 2 \% \text {, musc. } 2 \% \text { bio.; } \\
\text { traces of chalc., feldspar, } \\
\text { titanite. }\end{array}$} \\
\hline 17 & Sandstone, hematitic.. & & B & 7.5 & 1.5 & 9.4 & .... do ... & & \\
\hline 18 & Sandstone, limonitic. & & B & 9.4 & 8.7 & 10.4 & ......do. & & \\
\hline 19 & ....... do & ............... & B & 8.5 & 7.0 & 10.2 & ...... do & & \\
\hline 20 & ....... do & ...do do & B & 10.1 & 8.5 & 10.9 & .... do & & \\
\hline 21 & Sandstone, chloritic.. & ............... & B & 10.4 & 10.3 & 11.0 & ............. & & \\
\hline
\end{tabular}

See footnotes at end of table. 
TABLE 2.-Description, source, thermal-expansion coefficients, texture, and mineral composition of all materials tested-Continued.

\begin{tabular}{|c|c|c|c|c|c|c|c|c|c|}
\hline \multirow{2}{*}{$\begin{array}{l}\text { Speci- } \\
\text { men } \\
\text { num- } \\
\text { ber }\end{array}$} & \multirow{2}{*}{ Description } & \multirow{2}{*}{ Source } & \multirow{2}{*}{$\begin{array}{c}\text { Des- } \\
\text { igna- } \\
\text { tion } \\
\text { in } \\
\text { table } \\
1\end{array}$} & \multicolumn{3}{|c|}{$\begin{array}{l}\text { Mean coefficient of } \\
\text { linear thermal ex- } \\
\text { pansion per degree } \\
\mathrm{C} \times 10^{\circ}\end{array}$} & \multirow{2}{*}{ Texture s } & \multicolumn{2}{|c|}{$\begin{array}{c}\text { Mineral constituents b (in decreasing order of abundance; } \\
\text { percentages approximate only) }\end{array}$} \\
\hline & & & & $\begin{array}{l}-20^{\circ} \text { to } \\
+60^{\circ}\end{array}$ & $\begin{array}{l}0^{\circ} \text { to } \\
+60^{\circ}\end{array}$ & $\begin{array}{l}+60^{\circ} \\
\text { to } 0^{\circ}\end{array}$ & & Major, more than 3 percent & Minor, less than 3 percent \\
\hline 22 & Sandstone, calcareous felds- & Bill Williams gravel (Park- & $\mathrm{V}$ & 5.1 & 5.0 & 5.7 & Medium & Feldspar and quartz cemented & Microcline, limo., mt. \\
\hline 23 & Sandstone, cherty micaceous & $\begin{array}{l}\text { Kutras gravel (Shasta Dam), } \\
\text { Calif. }\end{array}$ & $\mathrm{x}$ & 6.4 & 5.0 & 9.7 & Fine.. & $\begin{array}{l}\text { Microq., sericite, quartz, kao- } \\
\text { lin. }\end{array}$ & $\begin{array}{l}\text { Graphitic matter, limo., mica, } \\
\text { chlorite. }\end{array}$ \\
\hline 24 & Sandstone, feldspathic. & do. & $\mathrm{x}$ & 4.4 & 2.4 & 6.3 & ...... do .. & Microq., feldspar (or., ab.), & Chlorite; traces leucoxene, \\
\hline $\begin{array}{l}25 \\
26\end{array}$ & do do & Cowlitz River gravel, Wash. & $\stackrel{\mathrm{Y}}{\mathrm{Y}}$ & $\begin{array}{l}4.8 \\
6.8\end{array}$ & $\begin{array}{l}3.5 \\
6.5\end{array}$ & $\begin{array}{l}6.7 \\
8.1\end{array}$ & $\begin{array}{l}\text { Medium.... } \\
\text { Fine...... }\end{array}$ & $\begin{array}{l}\text { Feldspar } 60 \% \text {. quartz } 35 \% \\
\text { Mieroq., feldspar, quartz, seri- } \\
\text { cite, chlorite or antigorite, }\end{array}$ & $\begin{array}{l}\text { Mica } 3 \% \text {, chlorite } 2 \% \text {, sericite. } \\
\text { Altered mica; trace of iron } \\
\text { oxides. }\end{array}$ \\
\hline 27 & $\ldots$ & $\begin{array}{l}\text { Kutras gravel (Shasta Dam), } \\
\text { Calif. }\end{array}$ & $\mathrm{x}$ & 7.9 & 7.4 & 9.1 & $\mathrm{Mec}$ & $\begin{array}{l}\text { graphite. } \\
\text { Quartz, feldspar, microq....... }\end{array}$ & Mica, chlorite, epidote. \\
\hline $\begin{array}{l}28 \\
29\end{array}$ & $\begin{array}{l}\text { Chalcedony } . . . . . \\
\text { Flint chat }\end{array}$ & $\begin{array}{l}\text { Serra do Mar, Brazil } \\
\text { Lead-zinc mine area, Mo }\end{array}$ & -... & $\begin{array}{l}13.8 \\
11.4\end{array}$ & $\begin{array}{l}13.8 \\
11.8\end{array}$ & $\begin{array}{l}14.2 \\
12.0\end{array}$ & $\begin{array}{l}\text { Fine } \\
\text { Verv fine.... }\end{array}$ & $\begin{array}{l}\text { Fibrous quartz } \\
\text { Microq., chalcedony.- }\end{array}$ & \\
\hline 30 & Chert, brown & $\begin{array}{l}\text { Potomac River gravel, Md.- } \\
\text { Va. }\end{array}$ & $\mathrm{B}$ & 11.2 & 11.4 & 11.4 & Very fine to fine. & Microq. $65 \%$, chalc. $35 \%$ & Limonite $1 \%$, trace of quartz. \\
\hline $\begin{array}{l}31 \\
32\end{array}$ & $\begin{array}{l}\text { Chert, white. } \\
\text { Chert, gray.- }\end{array}$ & $\begin{array}{l}\text { do } \\
\text { Silver Hill gravel, } \mathrm{M} d .\end{array}$ & $\stackrel{\mathrm{B}}{\mathrm{C}}$ & $\begin{array}{l}10.7 \\
11.3\end{array}$ & $\begin{array}{l}10.7 \\
11.6\end{array}$ & $\begin{array}{l}11.0 \\
11.6\end{array}$ & $\begin{array}{l}\text { Very fine } \\
\text { Very fine to fine... }\end{array}$ & $\begin{array}{l}\text { Microq., chalcedony } \\
\text { Microq. } 65 \% \text {, quartz } 35 \%\end{array}$ & (Rhombic holes.) \\
\hline 33 & Che & do & $\mathrm{C}$ & $\begin{array}{l}11.0 \\
11.2\end{array}$ & $\begin{array}{l}11.0 \\
11.4\end{array}$ & $\begin{array}{l}11.0 \\
11.6\end{array}$ & & Microcrystal & Chalcedony, (rh \\
\hline $\begin{array}{l}34 \\
35\end{array}$ & Chert, brown & Reading gravel, Mo & $\mathrm{T}$ & 11.4 & $\begin{array}{l}11.6 \\
10.8\end{array}$ & $\begin{array}{l}11.6 \\
11.4\end{array}$ & Very fine to fine... & Microq., quartz & Limonite, (porous). \\
\hline $\begin{array}{l}35 \\
36\end{array}$ & Chert, light gray. & Reading gravel, Mo & $\mathrm{T}$ & $\begin{array}{l}10.9 \\
11.5\end{array}$ & $\begin{array}{l}10.8 \\
11.7\end{array} \mid$ & $\begin{array}{l}11.4 \\
11.9\end{array}$ & $\begin{array}{l}\text { Very fine } \\
\text { Very fine }\end{array}$ & Microq., chalcedony.. & Limonite. \\
\hline $\begin{array}{l}37 \\
38\end{array}$ & $\begin{array}{l}\text { Chert, brown } \\
\text { Chert, white........ }\end{array}$ & Tishomingo gravel, Miss.... & $\begin{array}{l}\mathrm{T} \\
\mathrm{O}\end{array}$ & $\begin{array}{l}11.8 \\
11.0\end{array}$ & $\begin{array}{l}12.1 \\
11.2\end{array}$ & $\begin{array}{l}12.0 \\
11.4\end{array}$ & 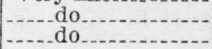 & $\begin{array}{l}\text { Microq., quartz } \\
\text { Microq., (various shaped }\end{array}$ & $\begin{array}{l}\text { Limonite, (porous). } \\
\text { Quartz. }\end{array}$ \\
\hline 39 & Chert, light gray & $\ldots$ do & 0 & 11.0 & 10.9 & 11.4 & .................. & $\begin{array}{l}\text { holes). } \\
\text { Mieroq., chale., (rhombic }\end{array}$ & Quartz veinlets. \\
\hline $\begin{array}{l}40 \\
41\end{array}$ & $\begin{array}{l}\text { Chert, brown } \\
\text { Chert, light-colored.... }\end{array}$ & Bank gravel, Kentucky & $\begin{array}{c}0 \\
\mathrm{~N}-1\end{array}$ & $\begin{array}{l}11.0 \\
11.4\end{array}$ & $\begin{array}{l}11.0 \\
11.4\end{array}$ & $\begin{array}{l}11.4 \\
11.9\end{array}$ & $\begin{array}{l}\text { Very fine, variable } \\
\text { do }\end{array}$ & $\begin{array}{l}\text { holes). } \\
\text { Microq., (rhombic holes), quartz } \\
\text { Microcrystalline quartz }\end{array}$ & $\begin{array}{l}\text { Limo., goethite; trace chalc. } \\
\text { (Rhombic holes), quartz. }\end{array}$ \\
\hline 42 & Chert, oolitic... & $\begin{array}{l}\text { Dam area, Tenn. } \\
\text { River gravel, Kentucky }\end{array}$ & $\mathrm{N}-2$ & 11.4 & 11.7 & 11.8 & Very fine to fine, & Microq., quartz... & Calcite, limonite. \\
\hline 43 & Chert, dark gray. & $\begin{array}{l}\text { Dam area, Tenn. } \\
\text { Cumberland River gravel, }\end{array}$ & $\mathrm{L}$ & 11.9 & 12.4 & 12.4 & $\begin{array}{l}\text { oolitic. } \\
\text { Very fine and } \\
\text { medium. }\end{array}$ & Chale. $65 \%$, microq. $35 \%$ & Cal., (rhombic holes): trace \\
\hline $\begin{array}{l}44 \\
45\end{array}$ & Chert, brown & $\begin{array}{l}\text { Tenn. } \\
\text { Estill Springs gravel, Tenn }\end{array}$ & $\stackrel{\mathrm{L}}{\mathrm{M}}$ & $\begin{array}{l}11.7 \\
11.8\end{array}$ & $\begin{array}{l}11.9 \\
12.0\end{array}$ & $\begin{array}{l}11.9 \\
12.3\end{array}$ & $\begin{array}{l}\text { medium. } \\
\text { Very fine } \\
\end{array}$ & $\begin{array}{l}\text { Chalc. } 50 \% \text {, microq. } 50 \% \text {. } \\
\text { Microcrystalline quartz_. }\end{array}$ & $\begin{array}{l}\text { limo. } \\
\text { Limo., (rhombic holes), quartz. } \\
\text { (Rhombic holes), limo., }\end{array}$ \\
\hline 46 & Chert, brown fossiliferous. & ......do.. & M & 11.4 & 11.8 & 11.5 & $\begin{array}{l}\text { Very fine to fine, } \\
\text { irregular. }\end{array}$ & Quartz, microq.. & $\begin{array}{l}\text { quartz. } \\
\text { Calcite, limonite, }\end{array}$ \\
\hline
\end{tabular}




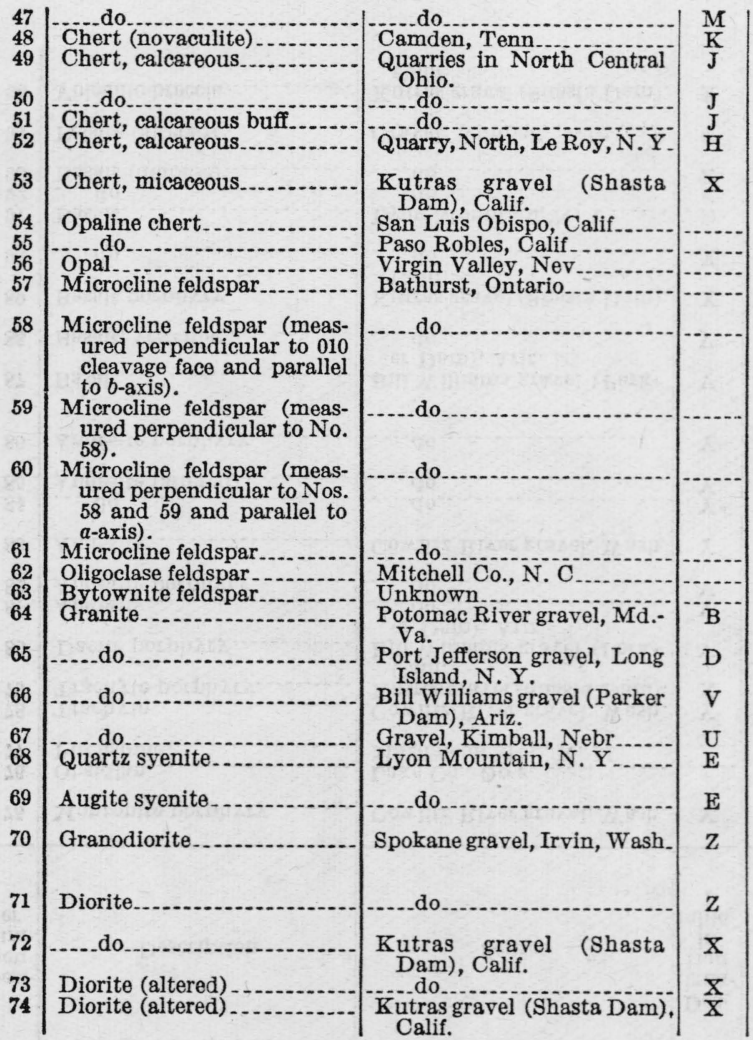

\begin{tabular}{|c|c|c|c|}
\hline $\begin{array}{l}\text { 11. } 2 \\
11.4 \\
10.6\end{array}$ & $\begin{array}{l}11.3 \\
11.6 \\
10.8\end{array}$ & $\begin{array}{l}11.6 \\
11.8 \\
10.9\end{array}$ & $\begin{array}{l}\text { Very fine, even } \\
\text { Very fine to fine }\end{array}$ \\
\hline & $\begin{array}{r}11.5 \\
9.3\end{array}$ & & $\ldots$ \\
\hline & 12.6 & & $\ldots$ \\
\hline 7.3 & 7.2 & 8.1 & Very fine... \\
\hline 4 & 14.1 & 14. 2 & Amo \\
\hline & 14. & 14. 6 & 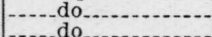 \\
\hline & 0.8 & 0.9 & Single twi \\
\hline 1.1 & 1.4 & 1.3 & ..do. \\
\hline 3.5 & 3.3 & 3. 2 & ...do. \\
\hline .1 & 17.3 & 17.3 & ...do... \\
\hline & & & 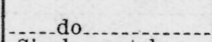 \\
\hline & 3. & & Singl \\
\hline & 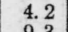 & & $\begin{array}{l}\text { Coar } \\
\text { Med }\end{array}$ \\
\hline & 9.3 & & Mediur \\
\hline & 5.8 & 6.8 & ....... do \\
\hline 1.8 & -1.7 & 4.6 & ... do \\
\hline 7. 2 & $\begin{array}{l}6.4 \\
7.3\end{array}$ & $\begin{array}{l}8.4 \\
7.7\end{array}$ & $\begin{array}{l}\text { Medit } \\
\text { Mediv }\end{array}$ \\
\hline & 5.8 & 6.1 & .....do. \\
\hline 3. 6 & 3.7 & 4.3 & ....... do. \\
\hline & 6.7 & 7 & . d \\
\hline & 5.6 & 6.9 & Medium to coars \\
\hline & 8 & 7.1 & $\begin{array}{l}\text { Med } \\
\text { Med }\end{array}$ \\
\hline
\end{tabular}

-do Microq. $85 \%$, rhombic holes)

Microq. $90 \%$, calcite $10 \%$ Micro

Microq., sericite.

Opal $90 \%$, chalcedony $10 \%$ Opal (relic diatom structures) Perthite (microcline $90 \%$, ab.

Similar to No. 57

Same crystal as No. 58

..do

Similar to Nos. 57 and $58 \ldots$ Bytownite feldpar $97 \%$

Or. perthite, microcine, quartz, ab.

Microcline, quartz, perthite,

Or., microcline, quartz, perth ite, ab.

Or., oligoclase, quartz

Microcline $45 \%$, perthite $20 \%$, ab. $20 \%$, quartz $10 \%$

$25 \%$ ab $5 \%$, $55 \%$, augite Pc. $50 \%$ quartz $25 \%$, or. $10 \%$ bio. $5 \%$, hb. $3 \%$.

Amphibole (hb. and/or actin-

Sericite, oligoclase, hb., chlorite. Zoisite, hb. and/or actinolite..-
Zoisite, hornblende, quartz...-
Cal., limo., (rhombic holes). Trace of iron oxide.

Quartz; trace of limonite. Limonite, quartz. Traces of quartz, matter.
Traces of epidote, actinolite.

Trace of iron oxide.

Quartz less than $1 / 2 \%$.

Muscovite, quartz. Garnet, chlorite, limo.

Sericite, biotite.

Biotite, sericite.

Musc., zoisite, sericite. Chlorite, cal., mt., augite Quartz, titanite.

Titanite, zoisite, chlorite, and sericite, each $2 \%$; traces of mt., apatite, cal.
Cal., zoisite, sericite, quartz, mt., titanite.
Mt., zoisite, epidote, limo. Quartz, titanite. 
TABLE 2.-Description, source, thermal-expansion coefficients, texture, and mineral composition of all materials tested-Continued.

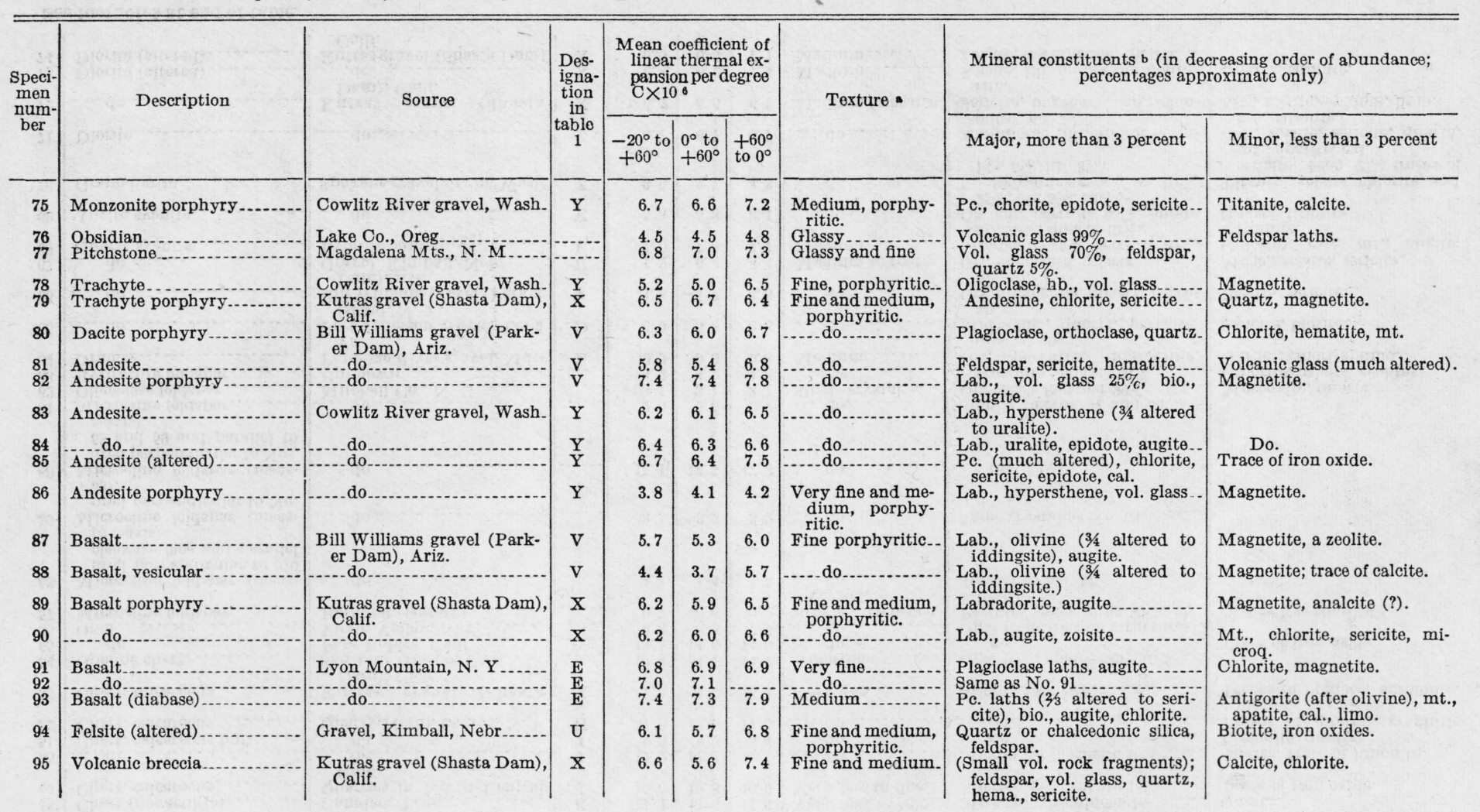




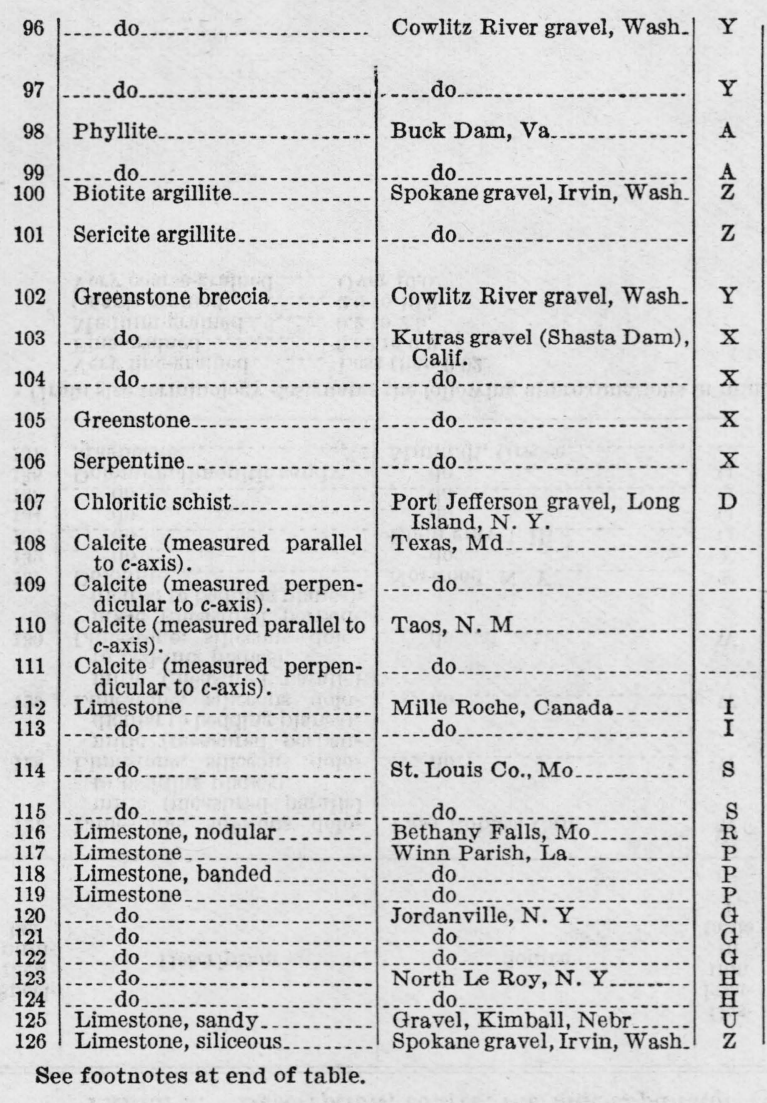

\begin{tabular}{|c|c|c|c|}
\hline .9 & 6.8 & 7.4 & $\begin{array}{l}\text { Very fine to fine } \\
\text { and very coarse. }\end{array}$ \\
\hline 6.1 & 6.2 & 8.0 & Very fine to \\
\hline 6.1 & 0.6 & 10.7 & \\
\hline & $\begin{array}{r}.1 \\
9.6\end{array}$ & .7 & Fine \\
\hline 9.8 & 9.8 & 10.1 & \\
\hline 5.1 & 5.4 & 8 & $\begin{array}{l}\text { Ver, } \\
\text { an }\end{array}$ \\
\hline 6. 6 & 6.6 & 7.5 & Fine \\
\hline 8.2 & 7.3 & 9 & \\
\hline 6.2 & 6.2 & 0 & Fin \\
\hline 6.0 & 6. & 9 & Fin \\
\hline 6.7 & & 5 & do \\
\hline 2.0 & 2 & 5 & ing \\
\hline 4.0 & -4.8 & -4.2 & ...... do _. \\
\hline 25.7 & 26 & 26.0 & Singl \\
\hline .0 & -4.8 & 8 & 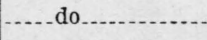 \\
\hline & & $\begin{array}{l}4.1 \\
4.0\end{array}$ & \\
\hline & & 4.4 & \\
\hline & $\begin{array}{l}5 . \\
3 .\end{array}$ & $\begin{array}{l}7.4 \\
4.3\end{array}$ & \\
\hline & 0 & $\begin{array}{l}-0.5 \\
-4\end{array}$ & Fin \\
\hline & & 9.0 & $\mathrm{Me}$ \\
\hline & $\begin{array}{l}4 . \\
5\end{array}$ & & \\
\hline & & & \\
\hline & & & \\
\hline & & t. & \\
\hline & & & \\
\hline
\end{tabular}

(Large vol. rock fragments); pc., epidote, actinolite, chloPc., quartz, chlorite, microq Quartz, microq. and chalc. sericite, calcite.

Quartz $45 \%$, sericite $35 \%$, bio.

Quartz $45 \%$, sericite $45 \%$, bio. (part altered to chlorite) Actinolite, microq., feldspar (altered).

Oligoclase, epidote, chlorite,

Feldspar, chlorite, epidote,

sericite.

Actinolite, quartz, epidote, Antigorite (intersecting lamellas).

Quartz $75 \%$, chlorite $15 \%$,

Same crystal as No. 108

Calcite.

Same crystal as No. 110 .......

Calcite $95 \%$, quartz $4 \%$ Similar to No. 112 ..

Calcite, microcrystalline quartz.

Calcite $98 \%$

C.....do

Calcite.

..... do.

Similar to No 120

Similar to No. 120

Calcite $99 \%$

Similar to No. 123

Calcite $50 \%$, quartz, feldspar

Calcite, quartz
Mt.; traces sericite, calcite. Sericite; traces mt., limo.

Micas, limo., feldspar; trace chlorite.

Titanite, leucoxene.

Musc.; traces py, graphitic matter.

Titanite, sericite, chlorite, magnetite.

Quartz.

Titanite, magnetite.

Hb. and/or olivine, py., tiMagnetite, feldspar.

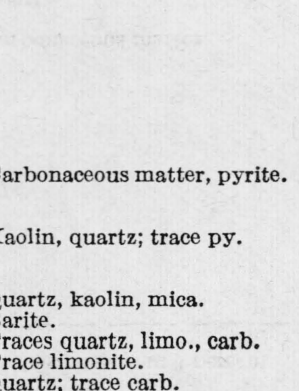

Quartz; trace carb.

Traces carb., kaolin, quartz.

Opal, mica.

Musc., limo., pyrite. 
TABLE 2.-Description, source, thermal-expansion coefficients, texture, and mineral composition of all materials tested-Continued

\begin{tabular}{|c|c|c|c|c|c|c|c|c|c|}
\hline \multirow{2}{*}{$\begin{array}{l}\text { Speci- } \\
\text { men } \\
\text { num- } \\
\text { ber }\end{array}$} & \multirow{2}{*}{ Description } & \multirow{2}{*}{ Source } & \multirow{2}{*}{$\begin{array}{c}\text { Des- } \\
\text { igna- } \\
\text { tion } \\
\text { in } \\
\text { table } \\
1\end{array}$} & \multicolumn{3}{|c|}{$\begin{array}{l}\text { Mean coefficient of } \\
\text { linear thermal ex- } \\
\text { pansion per degree } \\
\text { Cx10 }\end{array}$} & \multirow{2}{*}{ Texture a } & \multicolumn{2}{|c|}{$\begin{array}{c}\text { Mineral constituents b (in decreasing order of abundance; } \\
\text { percentages approximate only) }\end{array}$} \\
\hline & & & & $\begin{array}{c}-20^{\circ} \text { to } \\
+60^{\circ}\end{array}$ & $\begin{array}{l}0^{\circ} \text { to } \\
+60^{\circ}\end{array}$ & $\begin{array}{l}+60^{\circ} \\
\text { to } 0^{\circ}\end{array}$ & & Major, more than 3 percent & Minor, less than 3 percent \\
\hline 127 & $\begin{array}{l}\text { Limestone, siliceous dolo- } \\
\text { mitic (measured parallel } \\
\text { to bedding planes). }\end{array}$ & Paso Robles, Calif_- & $\mathrm{w}$ & 8.6 & 9.1 & 9.4 & ...... do... & $\begin{array}{l}\text { Dolomite and cal., opal } 10 \% \text {, } \\
\text { chalc. } 10 \% \text {. }\end{array}$ & \multirow{9}{*}{$\begin{array}{l}\text { Carbonaceous matter. } \\
\text { Quartz. } \\
\text { Limonite. } \\
\text { Musc., limo., feldspar. } \\
\text { Trace limonite. }\end{array}$} \\
\hline 128 & $\begin{array}{l}\text { Limestone, siliceous dolo- } \\
\text { mitic (measured perpen- } \\
\text { dicular to bedding planes). }\end{array}$ & .......... & $\mathrm{w}$ & 9.4 & 9.7 & 10.0 & .....do... & Same as No. 127 & \\
\hline 129 & $\begin{array}{l}\text { Limestone, siliceous dolo- } \\
\text { mitic (measured parallel } \\
\text { to bedding planes). }\end{array}$ & .......... & $\mathrm{w}$ & 8.9 & 9.2 & 9.3 & ............... & \multirow{7}{*}{$\begin{array}{l}\text { Similar to No. } 127 \\
\text { Same as No. } 129 \\
\text { Dolomite 95\%, quartz 5\% } \\
\text { Similar to No.131. } \\
\text { Dolomite. } \\
\text { do } \\
\text { Dolomite, quartz } 5 \% \\
\text { Dolomite } 50 \%, \\
\text { Magnesite, kaolin } 5 \% \\
50 \%\end{array}$} & \\
\hline 130 & $\begin{array}{l}\text { Limestone, siliceous dolo- } \\
\text { mitic (masured perpen- }\end{array}$ & do & $\mathrm{w}$ & 9.5 & 9.8 & 9.8 & ............... & & \\
\hline 131 & $\begin{array}{l}\text { dicular to bedding planes). } \\
\text { Dolomite }\end{array}$ & Norwood, N. Y.. & F & 6.6 & 5. 9 & 8.1 & Fine & & \\
\hline $\begin{array}{l}132 \\
133\end{array}$ & -....do do & Elgin gravel, III & ${ }_{Q}^{F}$ & $\begin{array}{l}7.6 \\
8.2\end{array}$ & $\begin{array}{l}7.7 \\
8.6\end{array}$ & 8.7 & do... do & & \\
\hline 134 & - & -...do do & $Q$ & 7.3 & 7.1 & 8.4 & .....do... & & \\
\hline 135 & do & -....do.... & Q & $\begin{array}{l}7.2 \\
8.7\end{array}$ & $\begin{array}{l}7.6 \\
8.5\end{array}$ & $\begin{array}{l}8.9 \\
9.3\end{array}$ & -...do do & & \\
\hline 137 & Magnesite & Muntodi, Greece & & 8.1 & 7.5 & 9.2 & Very fine... & & \\
\hline \multicolumn{2}{|c|}{$\begin{array}{r}\text { Grain } \\
\mathrm{Ve} \\
\mathrm{Fi} \\
\mathrm{M} \\
\mathrm{M} \\
\mathrm{C} \\
\mathrm{Ve} \\
\end{array}$} & $\begin{array}{l}\text { the following approximati } \\
\text { s than } 0.02 \text {. } \\
\text { to } 0.2 . \\
\text { to } 2.0 \text {. } \\
\text { to } 10.0 . \\
\text { er } 10.0 \text {. }\end{array}$ & in mill & limeters: & 328 & & 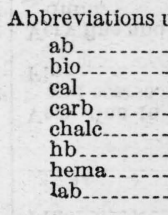 & $\begin{array}{l}\text { ite. } \\
\text { ite. } \\
\text { cite. } \\
\text { bonaceous matter. } \\
\text { lecedony. } \\
\text { nblende. } \\
\text { natite. } \\
\text { radorite. }\end{array}$ & \begin{tabular}{l} 
limonite. \\
microcrystalline quartz. \\
$0 .-$. muscovite. \\
magnetite. \\
orthoclase feldspar. \\
plagioclase feldspar. \\
\hdashline..-- pyrite. \\
\hdashline voleanic.
\end{tabular} \\
\hline
\end{tabular}




\section{RESULTS AND DISCUSSION}

The coefficients of linear thermal expansion of all materials tested are given for three different temperature ranges in table 2. Typical expansion curves of some of the materials are shown in figures 3 to 10 , inclusive. Variations in these coefficients for similar rocks or minerals may be attributed to three main factors, namely, crystal orientation, composition difference, and texture.

\section{EFFECT OF CRYSTAL ORIENTATION}

Measurements were made on oriented crystals of calcite (fig. 3), quartz (fig. 4), and microcline feldspar (fig. 5) to illustrate the differences in thermal expansivity that occur in minerals along different crystallographic axes. These values are given in table 3, together with values reported by other investigators. It can be seen, therefore, that with such minerals the thermal-expansion measurements on individual crystals of unknown orientation are of limited value.

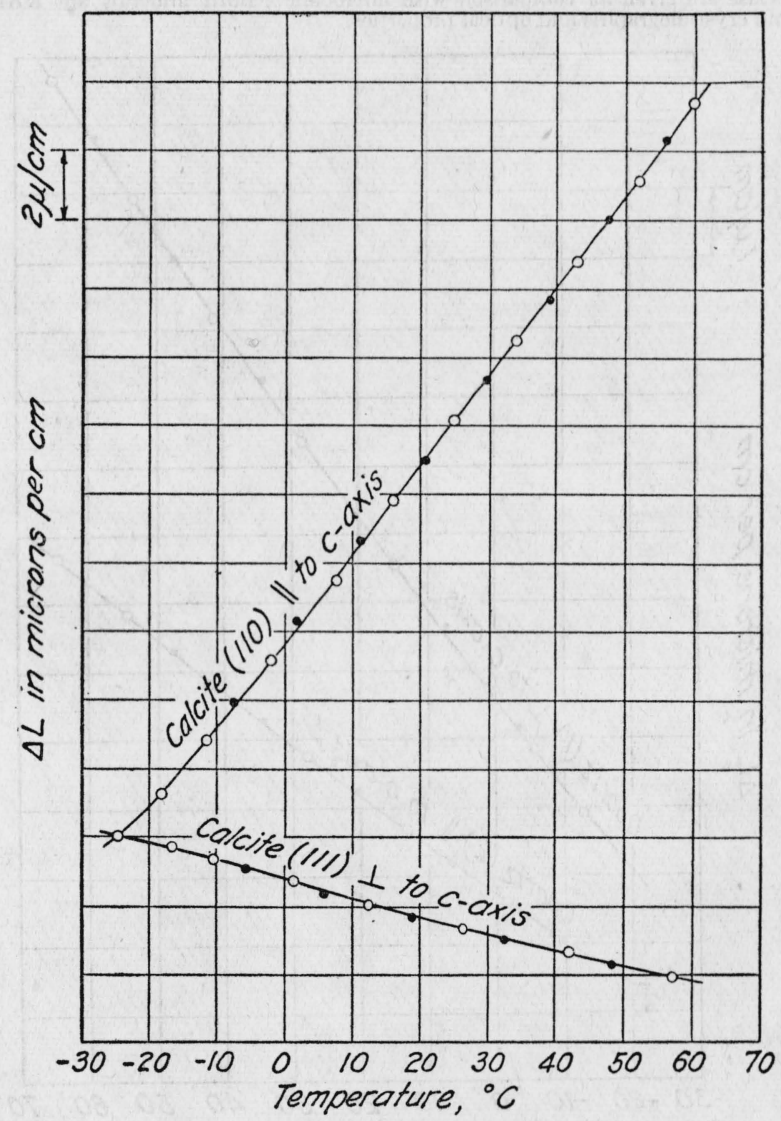

FIGURE 3.-Thermal-expansion curves of a crystal of calcite in two crystallographic directions.

Are heating points; are cooling points. Figures in parentheses refer to the specimen numbers in table 2. 


\section{Journal of Research of the National Bureau of Standards}

TABLE 3.-Mean coefficients of linear thermal expansion for oriented mineral specimens

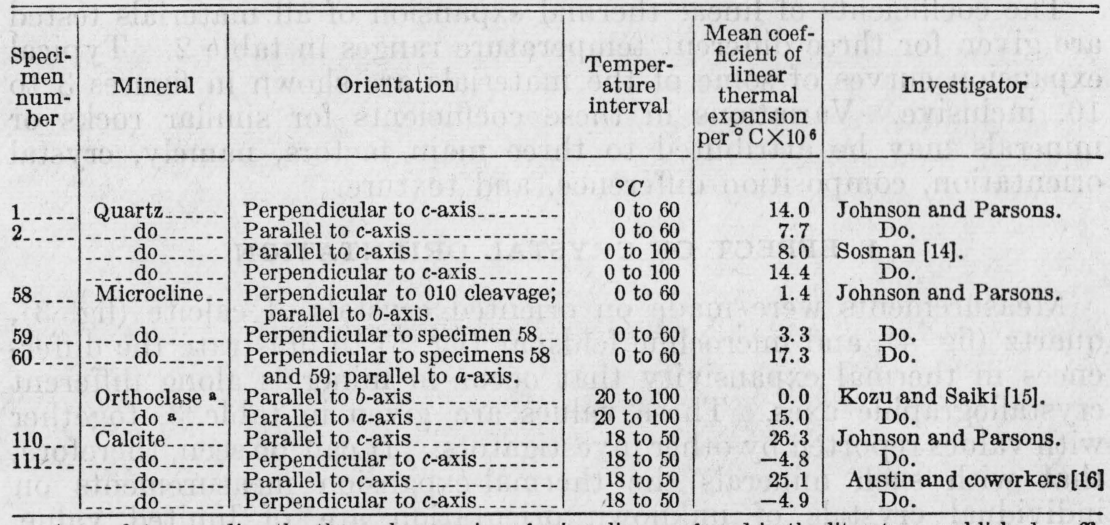

s As no data regarding the thermal expansion of microcline are found in the literature, published coefficients for orthoclase are given for comparison with microcline. Both minerals are $\mathrm{KAlSi}_{3} \mathrm{O}_{8}$, but vary slightly in certain crystallographic and optical properties.

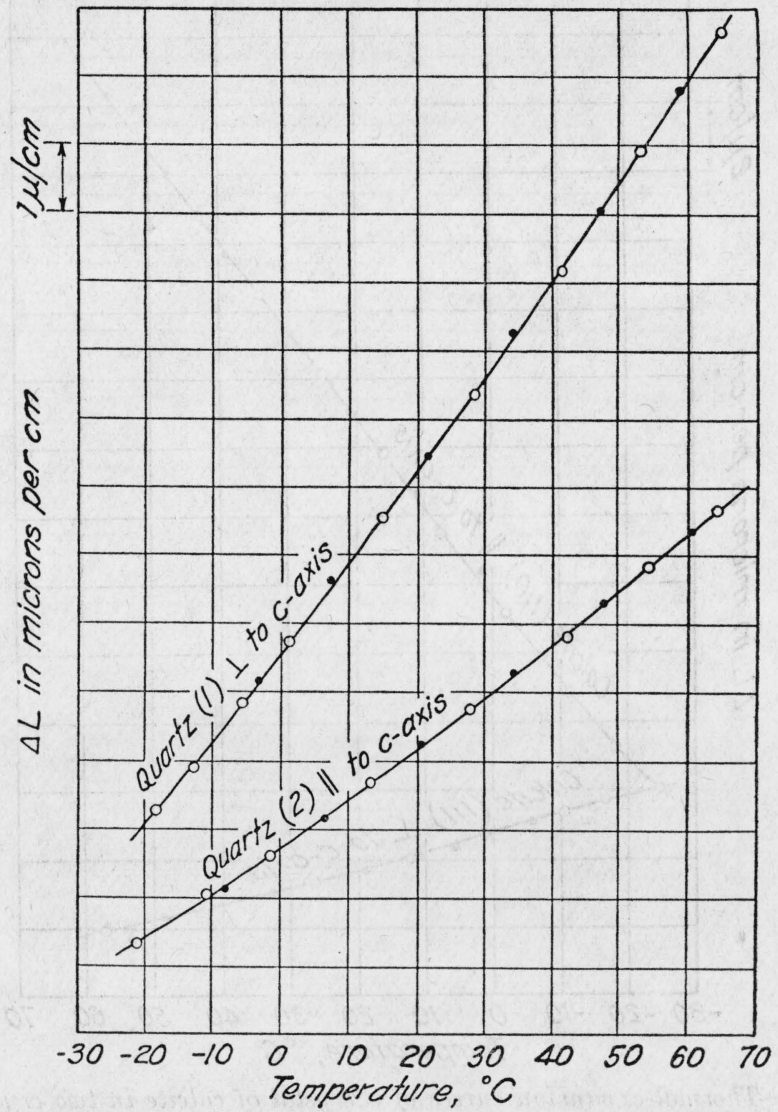

FIgURE 4.-Thermal-expansion curves of a crystal of quartz in two crystallographic directions.

Are heating points; are cocling points. Figures in parentheses refer to the specimen numbers in table 2. 
This is illustrated by specimen 62 (table 2), oligoclase feldspar, which shows a coefficient of $3.1 \times 10^{-6}$ per degree centigrade but whose orientation is unknown.

A monomineralic rock composed of many crystals with haphazard crystallographic orientations should have, in all directions, a thermalexpansion coefficient representing an average of all possible orientations of the mineral concerned. The 19 cherts, for example, which are composed almost entirely of very fine-grained quartz (specimens 30 to 48 , table 2 ) all have nearly equal thermal-expansion coefficients, averaging $11.3 \times 10^{-6}$ per degree centrigrade. This value is very close to the arithmetric average of the thermal expansion of quartz in all crystallographic directions, and, therefore, is representative of the coefficient of thermal expansion of rocks composed almost entirely of quartz grains with random orientations. If, on the other hand, a rock has more of its component crystal grains oriented in one direction than in any other, the coefficient of expansion in that direction will be different from those in other directions. An example of this is the siliceous dolomitic limestone from Paso Robles, Calif. (specimens 127 to 130,

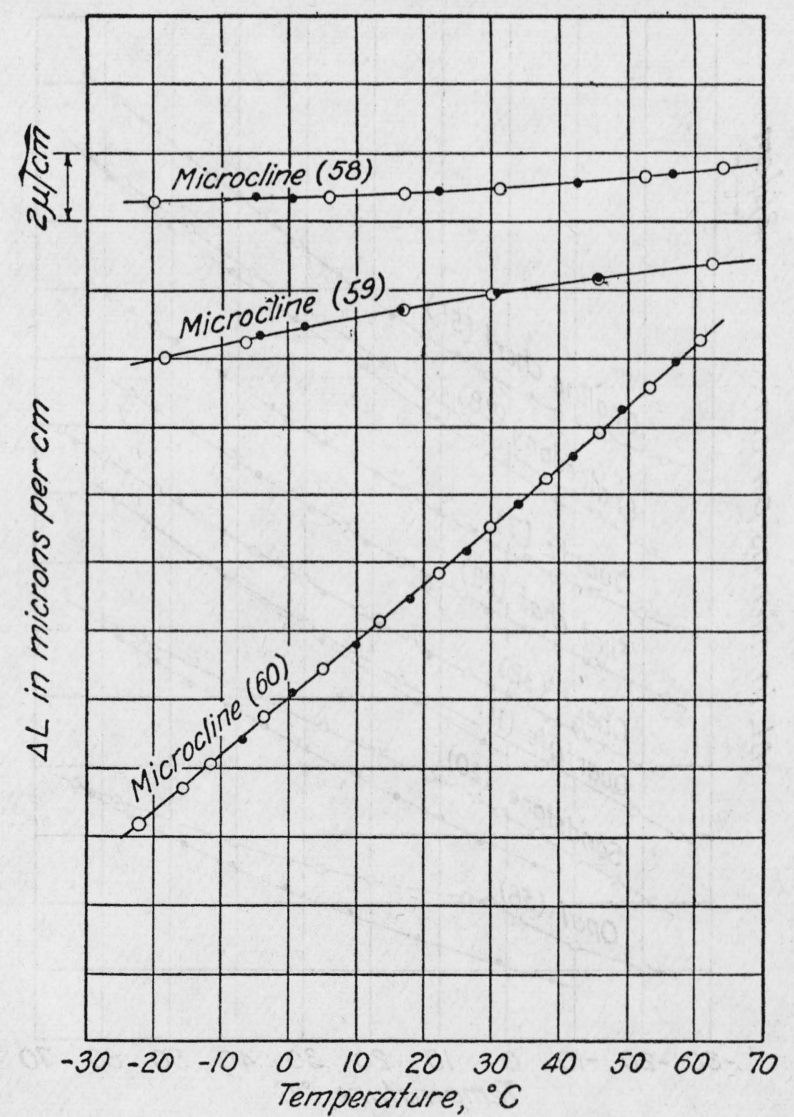

FIGURE 5.-Thermal-expansion curves of a crystal of microcline feldspar.

No. 58 perpendicular to 010 cleavage face and parallel to $b$-axis; No. 59 , perpendicular to No. 58 ; No. 60 , perpendicular to Nos. 58 and 59 and parallel to $a$-axis. $O$ Are heating points; 9 are cooling points; figures in parentheses refer to specimen numbers in table 2 . 
table 2), which has a coefficient of $8.7 \times 10^{-6}$ parallel to its bedding planes and $9.4 \times 10^{-6}$ perpendicular to its bedding planes. Another suspected example is the limestone from Jordanville, N. Y. Three specimens of this limestone taken in random directions gave coefficients of $4.0,4.8$, and $9.1 \times 10^{-6}$ (Nos. 120,121 , and 122).

\section{EFFECT OF COMPOSITION DIFFERENCE}

Rocks from various sources but of similar composition have approximately the same expansivities, if orientation differences are disregarded. This is true for the siliceous rocks (fig. 6), the calcitic limestones (fig. 7), and the dolomites (fig. 7). As a specific example, cherts 30 to 48 (table 2), which were composed of little but silica, had notably similar coefficients ranging from 10.7 to $11.9 \times 10^{-6}$. When other minerals were present, however, as in Nos. 49 to 53, which contained varying amounts of calcite and mica (sericite), the coefficients ranged from 7.3 to $11.9 \times 10^{-6}$. Compare also sandstones 16 and 22 (fig. 8) and limestones 112 and 129 (fig. 8).

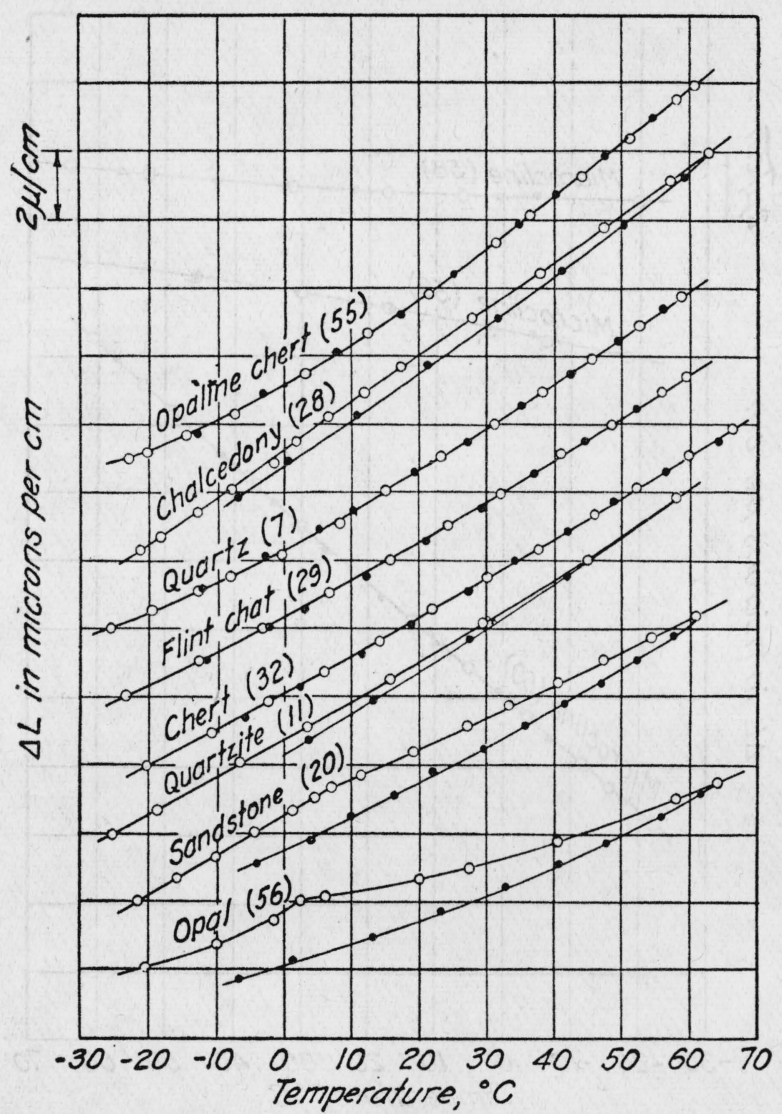

FIGURE 6.-Thermal-expansionicurves of various siliceous rocks.

$\mathrm{O}$ are heating points; are cooling points. Figures in parentheses refer to the specimen numbers in table 2. 


\section{EFFECT OF TEXTURE}

The kind of texture here considered is grain size, although other textures, such as crystallinity or noncrystallinity, may have important effects on rock expansivities. The grain size is particularly important in the present method of test because of the small size of the test specimen. The average height of the latter was only about 0.25 in., and the expansion of only one spacer was measured. The total number of grains through which measurement was made is strictly a function of the grain size of the rock and, based on the terminology given in section IV, was estimated to be as follows:

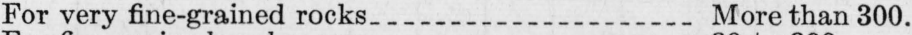

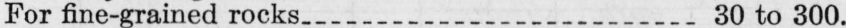

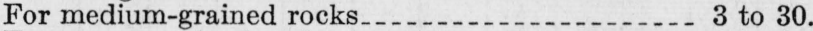
For coarse-grained rocks...................... 1 to 3 .
For very coarse-grained rocks.......... Less than 1.

The thermal-expansion coefficient of a specimen of very fine-grained, randomly oriented rock is, therefore, an average of all possible crystal

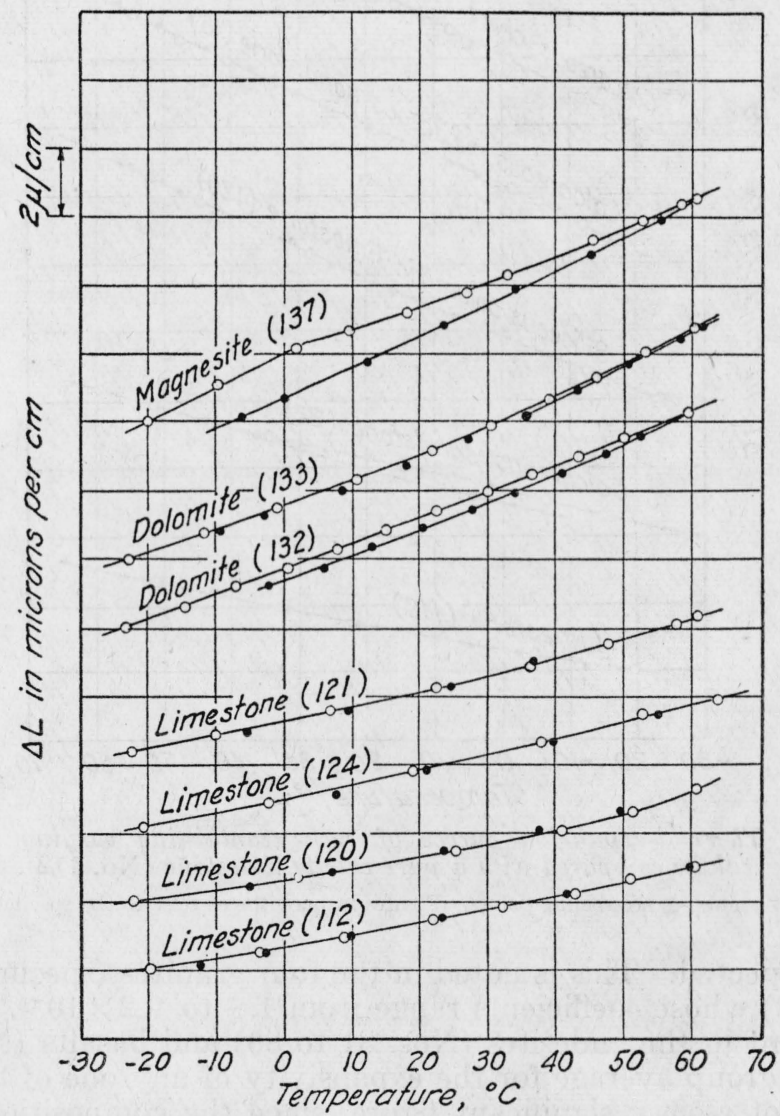

FIGURE 7.-Thermal-expansion curves of various carbonate rocks. 
orientations of all minerals in that rock and represents the actual expansivity of the rock. The coefficient given for a coarse-grained rock, however, may actually represent the expansivity of only one crystal orientation of one mineral and accordingly, cannot be considered as a true representation of the expansivity of that entire rock.

In the igneous and volcanic rocks, which have several minerals as part of their essential compositions and a large number of possible accessory and alteration minerals, considerable variation in expansivity

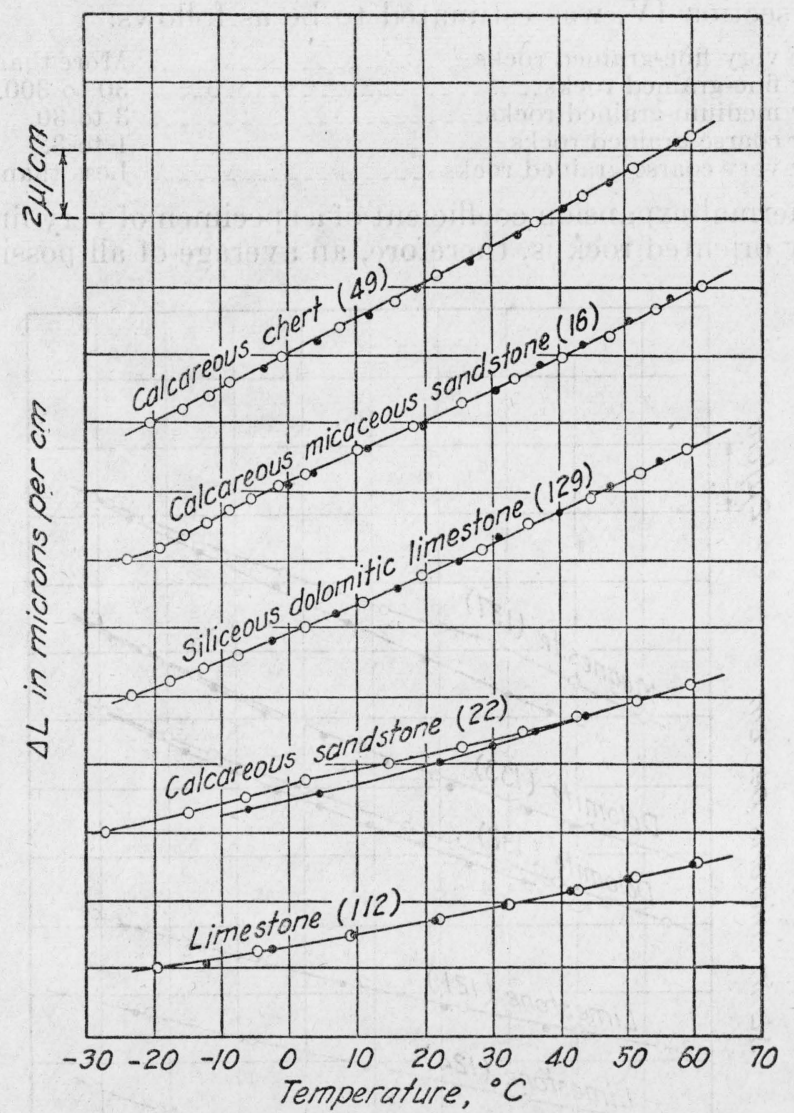

FIGURE 8.-Thermal-expansion curves of rocks containing varying amounts of calcite compared with a pure calcite limestone (No. 112).

Are heating points; are cooling points. Figures in parentheses refer to the specimen numbers in table 2 .

is to be expected. This is noted in the four granites (specimens 64 to 67 , table 2) whose coefficients range from 1.8 to $9.2 \times 10^{-6}$, and to a lesser extent in the andesites (Nos. 81 to 86 ) and basalts (Nos. 87 to 93). The group average for the expansivity of any one of these rock types is not a very significant figure, since the composition of such rocks is not necessarily as constant as the name suggests.

Also, different specimens of a particular mineral may differ in composition; for example, the combined water in opal varies in diff- 
erent opal specimens. This may help to explain the expansivity differences (table 2 and fig. 6 ) between Nevada opal (No. 56, coefficient $6.3 \times 10^{-6}$ ) and California opaline chert (specimens 54 to 55 , coefficients 13.4 and $13.3 \times\left(0^{-6}\right) .^{2}$

\section{MATERIALS WITH IRREGULAR EXPANSION}

Some materials do not expand uniformly on heating and the resulting expansion curves show more or less definite breaks in their con-

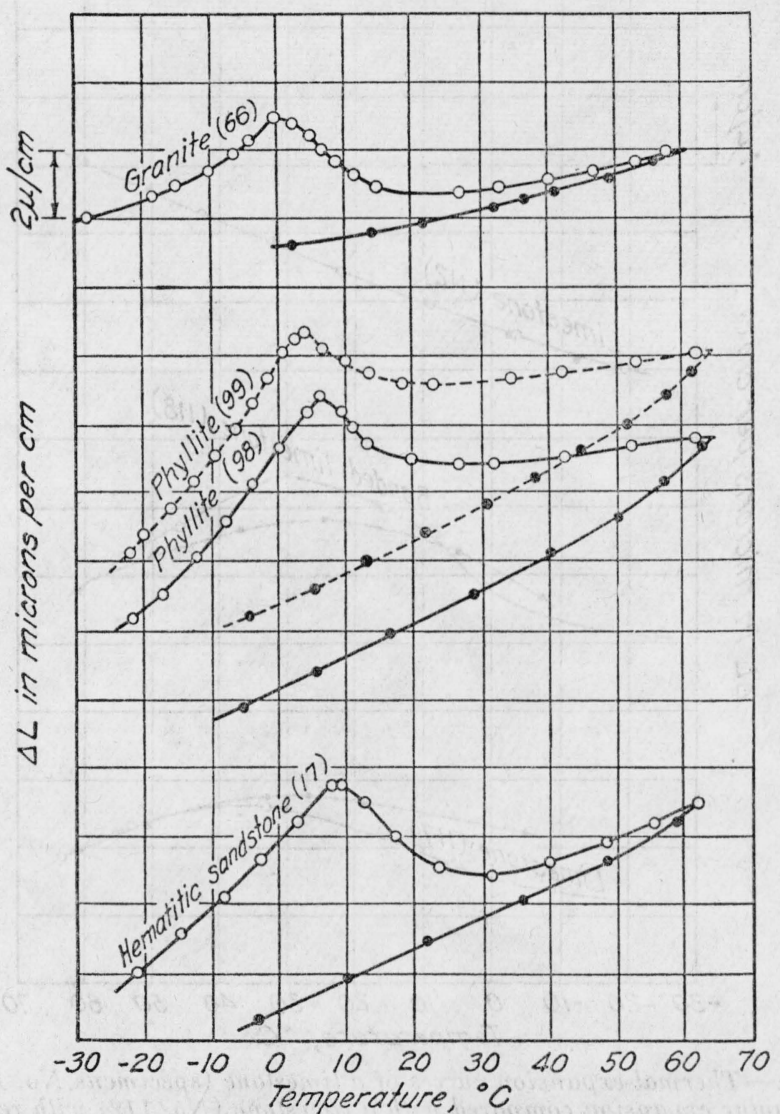

FIGURE 9.-Thermal-expansion curves of rocks with irregular expansion.

Nos. 98 and 99 are different specimens of the same rock sample; No. 66 was measured twice and No. 17 four times, and identical curves, respectively, were obtained each time: $O$ are heating points; are cooling points. Figures in parentheses identify specimens in table 2.

tinuity because of a change in the rate or a change in the direction of expansion. In most of these cases the materials expand on heating until temperatures between $0^{\circ}$ and $10^{\circ} \mathrm{C}$ have been reached. With further heating they either expand at a lesser rate (opal, No. 56, and sandstone 20, fig. 6) or contract (fig. 9) until temperatures between $15^{\circ}$ and $30^{\circ} \mathrm{C}$ have been reached, when expansion is resumed. As a

${ }^{2}$ Nevada opal showed an ignition loss at $1,300^{\circ} \mathrm{C}$ of 5.4 percent and the opal of the California opaline chert an ignition loss of 7.3 percent. 
general rule, these irregularities are not evident on cooling. Two specimens of a Louisiana limestone, however, were found that exhibited reversals both on heating and on cooling (fig. 10). The specimens exhibiting irregular expansion were retested with similar results; for example, specimen 66 (fig. 9) was measured by both the visual and the photographic methods, and specimen 17 (fig. 9) was tested twice by both methods. Identical curves were obtained, respec-

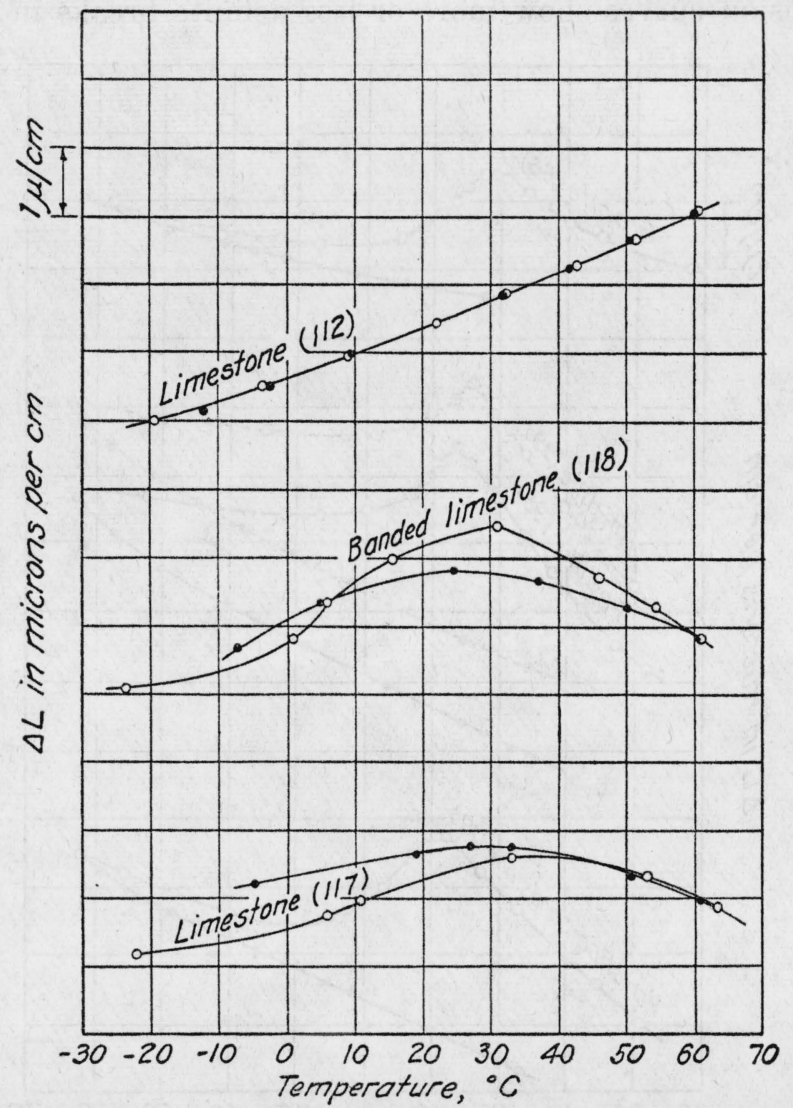

FIGURE 10.-Thermal-expansion curves of a limestone (specimens No. 117 and 118) with irregular expansion compared with a limestone (No. 112) with regular expansion.

are heating points; are cooling points. Figures in parentheses identify the specimens in table 2.

tively, for both specimens when retested. The coefficients given in table 2 inadequately describe the thermal-expansion behavior of these materials. For this reason, table 4 has been prepared to give the average thermal coefficients between those points where sudden changes in rates of expansion occurred.

It is possible that these irregularities are caused by capillary water in the specimens. The specimens were dried for several days over $\mathrm{CaCl}_{2}$, but all water was not necessarily removed. The specimens were held overnight at temperatures well below freezing, so that some 
of the capillary water present was undoubtedly frozen. As the ice melted, two complicating factors affected the rate of expansion of the specimens, namely (1) the volume change occurring as ice melts into the corresponding smaller volume of water, and (2) the change in heating rate of the specimen as the heat of crystallization of the water is absorbed. The latter factor may help to explain the occurrence of the breaks in continuity a few degrees above $0^{\circ} \mathrm{C}$. Additional

TABLE 4.-Mean coefficients of linear thermal expansion, between points of inflection, of materials having irregular expansions

\begin{tabular}{|c|c|c|c|c|c|c|c|}
\hline $\begin{array}{l}\text { Spec- } \\
\text { imen } \\
\text { num- } \\
\text { ber }\end{array}$ & Material & $\begin{array}{l}\text { Temper- } \\
\text { ature } \\
\text { interval }\end{array}$ & $\begin{array}{l}\text { Mean coef- } \\
\text { ficient of } \\
\text { linear } \\
\text { thermal } \\
\text { expansion } \\
\text { per }{ }^{\circ} \mathrm{C} \times 10^{6}\end{array}$ & $\begin{array}{l}\text { Spec- } \\
\text { imen } \\
\text { num- } \\
\text { ber }\end{array}$ & Material & $\begin{array}{l}\text { Temper- } \\
\text { ature } \\
\text { interval }\end{array}$ & $\begin{array}{c}\text { Mean coef- } \\
\text { ficient of } \\
\text { linear } \\
\text { thermal } \\
\text { expansion } \\
\text { per }{ }^{\circ} \mathrm{C} \times 10^{\circ}\end{array}$ \\
\hline $\begin{array}{l}17 \ldots \\
17 \ldots \\
17 \ldots\end{array}$ & \begin{tabular}{|c} 
Hematitic sandstone \\
\hdashline do \\
\end{tabular} & $\begin{array}{r}\circ C \\
-20 \text { to } 8 \\
8 \text { to } 30 \\
30 \text { to } 60\end{array}$ & $\begin{array}{r}18.8 \\
-12.9 \\
6.4\end{array}$ & $\begin{array}{l}98 \ldots \ldots \\
98 \ldots \ldots \\
98 \ldots \ldots\end{array}$ & 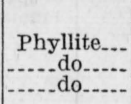 & $\begin{array}{c}\circ C \\
-20 \text { to } 7 \\
7 \text { to } 30 \\
30 \text { to } 60\end{array}$ & $\begin{array}{r}23.4 \\
-8.4 \\
2.4\end{array}$ \\
\hline $\begin{array}{l}19 \ldots \ldots \\
19 \\
19 \ldots\end{array}$ & \begin{tabular}{|l} 
Limonitic sandstone \\
\hdashline do \\
do \\
\end{tabular} & $\begin{array}{r}-20 \text { to } 6 \\
6 \text { to } 17 \\
17 \text { to } 60\end{array}$ & $\begin{array}{r}13.6 \\
-4.0 \\
9.2\end{array}$ & $\begin{array}{l}99 \ldots \\
99 \ldots \\
99 \ldots\end{array}$ & 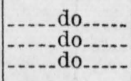 & $\begin{array}{r}-20 \text { to } 4 \\
4 \text { to } 23 \\
23 \text { to } 60\end{array}$ & $\begin{array}{r}24.8 \\
-8.0 \\
3.3\end{array}$ \\
\hline 23. & Argillaceous sandstone & $\begin{array}{r}-20 \text { to } 15 \\
15 \text { to } 60\end{array}$ & $\begin{array}{r}10.5 \\
3.2 \\
4.2\end{array}$ & $\begin{array}{l}115 \\
115 \\
115\end{array}$ & Limestone_- & $\begin{array}{r}-20 \text { to } 4 \\
4 \text { to } 20 \\
20 \text { to } 60\end{array}$ & $\begin{array}{l}9.2 \\
2.1 \\
6.4\end{array}$ \\
\hline $\begin{array}{l}24 \ldots \ldots \\
24 \ldots \ldots \\
24 \ldots \ldots\end{array}$ & \begin{tabular}{|c} 
Feldspathic sandstone.. \\
do
\end{tabular} & $\begin{array}{r}-20 \text { to } 6 \\
6 \text { to } 26 \\
26 \text { to } 60\end{array}$ & $\begin{array}{r}9.7 \\
-3.4 \\
4.9\end{array}$ & 11 & $\ldots$ & $\begin{array}{r}20 \text { to } 60 \\
-20 \text { to } 35 \\
35 \text { to } 60\end{array}$ & $\begin{array}{r}2.3 \\
-2.3\end{array}$ \\
\hline 25 & 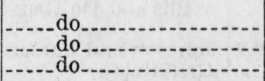 & $\begin{array}{r}-20 \text { to } 6 \\
6 \text { to } 18 \\
18 \text { to } 60\end{array}$ & $\begin{array}{r}9.5 \\
-2.2 \\
3.9\end{array}$ & & $\cdots-d$ & $\begin{array}{r}60 \text { to } 30 \\
30 \text { to } 0 \\
-20 \text { to } 31\end{array}$ & -2. \\
\hline 56. & Opal & $\begin{array}{r}-20 \text { to } 2 \\
2 \text { to } 60\end{array}$ & $\begin{array}{l}8.5 \\
5.4\end{array}$ & $\begin{array}{l}118 \ldots \\
118 . \ldots \\
118 . .\end{array}$ & 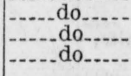 & $\begin{array}{l}31 \text { to } 60 \\
60 \text { to } 25 \\
25 \text { to } 0\end{array}$ & $\begin{array}{r}-5.4 \\
-2.8 \\
3.0\end{array}$ \\
\hline $\begin{array}{l}66 \ldots \\
66 \ldots \ldots \\
66 \ldots \ldots\end{array}$ & $\begin{array}{l}\text { Granite } \\
\text { do }\end{array}$ & $\begin{array}{r}-20 \text { to }-1 \\
-1 \text { to } 22 \\
22 \text { to } 60\end{array}$ & $\begin{array}{r}12.5 \\
-10.6 \\
3.2\end{array}$ & $\begin{array}{l}119 \ldots \\
119 \ldots\end{array}$ & $\ldots d$ & $\begin{array}{r}-20 \text { to } 25 \\
25 \text { to } 60\end{array}$ & $\begin{array}{r}3.9 \\
10.7\end{array}$ \\
\hline & - & $\begin{array}{r}-20 \text { to } 4 \\
4 \text { to } 15 \\
15 \text { to } 60\end{array}$ & $\begin{array}{r}10.5 \\
-1.5 \\
8.0\end{array}$ & $\begin{array}{l}131 \ldots \\
131 \ldots \\
131 \ldots\end{array}$ & 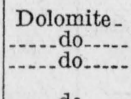 & $\begin{array}{r}-20 \text { to } 4 \\
4 \text { to } 7 \\
7 \text { to } 60\end{array}$ & $\begin{array}{r}8.2 \\
-1.1 \\
6.7\end{array}$ \\
\hline $\begin{array}{l}95 .- \\
95 \ldots\end{array}$ & $\begin{array}{l}\text { Volcanic breccia... } \\
- \text { do }\end{array}$ & $\begin{array}{r}-20 \text { to } 7 \\
7 \text { to } 60\end{array}$ & $\begin{array}{r}10.1 \\
4.7\end{array}$ & $\begin{array}{l}134 \ldots \\
134 \ldots \\
134 \ldots\end{array}$ & 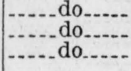 & $\begin{array}{r}-20 \text { to } 6 \\
6 \text { to } 11 \\
11 \text { to } 60\end{array}$ & $\begin{array}{r}7.5 \\
-1.8 \\
8.2\end{array}$ \\
\hline 97 & $\begin{array}{l}. . \text { do } \\
\text { do } \\
\text { do }\end{array}$ & $\begin{array}{r}-20 \text { to } 13 \\
13 \text { to } 15 \\
15 \text { to } 60\end{array}$ & $\begin{array}{r}5.7 \\
-4.0 \\
7.0\end{array}$ & & & & \\
\hline
\end{tabular}

study is needed on the effects of capillary water in the various materials. The irregular expansion of the limestone from Winn Parish, La., (specimens 117 and 118, table 4 and fig. 10) does not seem explainable as a water phenomenon, but no other explanation for these curves has yet been suggested.

\section{COMPARISON OF PRESENT RESULTS WITH PREVIOUS WORK}

The average coefficients for groups of similar rocks, as determined in the present study, are compared in table 5 with the results of other recent investigations. Not only are there variations in the average coefficients reported by the several investigators, but there are also considerable differences in values for individual specimens of the same 
rock type as determined in the present investigation (see range of coefficients, table 5). Although most of the average values obtained by other investigators differ from the averages given in the present paper, they do fall within or close to the range of values obtained for individual specimens in this study. The lack of agreement may be due to differences in methods of investigations, in temperature ranges, in crystal orientations, and in mineral composition and textures of the rocks used by the various investigators.

TABLE 5.-Mean coefficients of linear thermal expansion of similar materials determined by other invesigators and compared with the results of the present study

\begin{tabular}{|c|c|c|c|c|c|}
\hline \multirow[t]{2}{*}{ Material } & \multirow{2}{*}{$\begin{array}{l}\text { Number } \\
\text { of } \\
\text { of speci- } \\
\text { mens }\end{array}$} & \multirow{2}{*}{$\begin{array}{l}\text { Tempera- } \\
\text { ture } \\
\text { range }\end{array}$} & \multicolumn{2}{|c|}{$\begin{array}{l}\text { Mean coefficients of } \\
\text { linear thermal ex- } \\
\text { pansion per } \\
{ }^{\circ} \mathrm{C} \times 10^{\circ}\end{array}$} & \multirow[t]{2}{*}{ Investigator } \\
\hline & & & Average & Range & \\
\hline $\begin{array}{r}\text { Quartzite... } \\
\text { Do..... } \\
\text { Do..... }\end{array}$ & $\begin{array}{l}9 \\
3 \\
5\end{array}$ & $\begin{array}{l}{ }^{\circ} C \\
-20 \text { to }+60 \\
20 \text { to } 100 \\
0 \text { to } 60\end{array}$ & $\begin{array}{r}9.9 \\
10.6 \\
11.7\end{array}$ & 7.0 to 12.2 & $\begin{array}{l}\text { Johnson and Parsons. } \\
\text { Griffith [4]. } \\
\text { Willis and De Reus [5]. }\end{array}$ \\
\hline $\begin{array}{l}\text { Sandstone } \\
\text { Do } \\
\text { Do }\end{array}$ & $\begin{array}{r}11 \\
10 \\
\times 5\end{array}$ & $\begin{array}{r}-20 \text { to }+60 \\
20 \text { to } 100 \\
0 \text { to } 60\end{array}$ & $\begin{array}{r}7.4 \\
9.8 \\
11.7\end{array}$ & 4.4 to 10.4 & $\begin{array}{l}\text { Johnson and Parsons. } \\
\text { Griffith [4]. } \\
\text { Willis and De Reus [5]. }\end{array}$ \\
\hline $\begin{array}{l}\text { Chert } \\
\text { Do } \\
\text { Do }\end{array}$ & $\begin{array}{r}24 \\
1 \\
22\end{array}$ & $\begin{array}{r}-20 \text { to }+60 \\
20 \text { to } 100 \\
0 \text { to } 60\end{array}$ & $\begin{array}{l}11.1 \\
11.5 \\
12.2\end{array}$ & 7.3 to 11.9 & $\begin{array}{l}\text { Johnson and Parsons. } \\
\text { Griffith [4]. } \\
\text { Willis and De Reus [5]. }\end{array}$ \\
\hline $\begin{array}{l}\text { Granite } \\
\text { Do Do } \\
\text { Other granitic rocks: syenite, } \\
\text { diorite, monzonite. }\end{array}$ & $\begin{array}{r}4 \\
17 \\
2 \\
8\end{array}$ & $\begin{array}{r}-20 \text { to }+60 \\
20 \text { to } 100 \\
0 \text { to } 60 \\
-20 \text { to }+60\end{array}$ & $\begin{array}{l}6.1 \\
7.9 \\
5.5 \\
6.1\end{array}$ & $\mid \begin{array}{c}1.8 \text { to } 9.2 \\
3.6 \text { to } 7.0\end{array}$ & $\begin{array}{l}\text { Johnson and Parsons. } \\
\text { Griffith [4]. } \\
\text { Willis and De Reus [5]. } \\
\text { Johnson and Parsons. }\end{array}$ \\
\hline $\begin{array}{l}\text { Basalt. } \\
\text { Do } \\
\text { Other felsitic rocks: andesite, } \\
\text { dacite, trachyte. }\end{array}$ & $\begin{array}{r}7 \\
4 \\
2 \\
10\end{array}$ & $\begin{array}{r}-20 \text { to }+60 \\
20 \text { to } 100 \\
0 \text { to } 60 \\
-20 \text { to }+60\end{array}$ & $\begin{array}{l}6.2 \\
4.8 \\
9.4 \\
6.0\end{array}$ & \begin{tabular}{|c|}
4.4 to 7.4 \\
3.8 to 7.4
\end{tabular} & $\begin{array}{l}\text { Do. } \\
\text { Griffith [4]. } \\
\text { Willis and De Reus [5]. } \\
\text { Johnson and Parsons. }\end{array}$ \\
\hline $\begin{array}{l}\text { Limestone } \\
\text { Do } \\
\text { Do } \\
\text { Do } \\
\text { Do } \\
\text { Marble b } \\
\text { Do. b } \\
\text { Do }\end{array}$ & $\begin{array}{r}13 \\
22 \\
5 \\
16 \\
5 \\
2 \\
2 \\
9 \\
9 \\
6\end{array}$ & $\begin{array}{r}-20 \text { to }+60 \\
20 \text { to } 100 \\
0 \text { to } 20 \\
0 \text { to } 60 \\
-7 \text { to }+88 \\
-32 \text { to }+24 \\
24 \text { to } 100 \\
24 \text { to } 100 \\
-20 \text { to }+60\end{array}$ & $\begin{array}{r}4.4 \\
7.9 \\
4.6 \\
4.6 \\
7.6 \\
1.2 \\
7.8 \\
11.3 \\
8.3\end{array}$ & \begin{tabular}{|c|}
0.9 to 9.1 \\
3.6 to 9.8 \\
\end{tabular} & $\begin{array}{l}\text { Do. } \\
\text { Griffith [4]. } \\
\text { Do. } \\
\text { Willis and De Reus [5]. } \\
\text { Koenitzer [6]. } \\
\text { Pearson [1]. } \\
\text { Do. } \\
\text { Souder and Hidnert [7]. } \\
\text { Johnson and Parsons. }\end{array}$ \\
\hline $\begin{array}{l}\text { Dolomite } \\
\text { Dolomitic marble }\end{array}$ & $\begin{array}{l}6 \\
1\end{array}$ & $\begin{array}{l}-20 \text { to }+60 \\
-17 \text { to }+49\end{array}$ & $\begin{array}{l}7.6 \\
3.6\end{array}$ & 6.6 to 8.7 & $\begin{array}{l}\text { Do. } \\
\text { Pearson [1]. }\end{array}$ \\
\hline
\end{tabular}

a Measurements made on water-saturated specimens.

b Same specimens.

\section{AGGREGATE EXPANSIVITY IN RELATION TO CONCRETE}

The coefficient of linear thermal expansion of hardened portland cement varies considerably with such factors as the kind and composition of the cement, the water-cement ratio, and the age [8]. This coefficient varies within the normal range of atmospheric temperatures between limits of approximately $11 \times 10^{-6}$ and $16 \times 10^{-6}$ per degree centigrade ( 6 and $9 \times 10^{-6}$ per degree Fahrenheit), according to Davis [8], Pearson [1], and others [9]. As pointed out in the introduction, large differences in coefficients between the cementitious 
material and the aggregate may lead to concrete failure where severe weather conditions are encountered.

The discussion in section $\mathrm{V}, 1$ to 4 , indicates four types of materials, classified according to expansivity characteristics, which might manifest themselves unfavorably in concrete. These are (a) materials having a uniform coefficient of thermal expansion markedly different from the normal value for concrete; (b) an aggregate composed of single crystal fragments with different expansivity in various crystallographic directions; (c) an aggregate (a gravel or a crushed coarsegrained rock) composed of two or more fractions which are present in considerable amounts and have widely different expansivities; and (d) a material with irregular expansion of the type illustrated in figures 9 and 10. At least two instances of disintegration of concrete have been ascribed recently to type (a), namely: a dolomitic marble aggregate with a thermal coefficient of $3.6 \times 10^{-6}$ per degree centigrade studied by Pearson [1] and a fine-grained limestone aggregate with a coefficient of $4.0 \times 10^{-6}$ per degree centigrade mentioned by Hornibrook [2]. These aggregates were used in concretes that disintegrated after exposure to one northern United States winter. An example of (b) has been pointed out in Central New York by Swayze [17], where a coarse aggregate consisting of single calcite crystals was used in a cast stone. This stone was in very bad condition after exposure to one winter. The Kimball, Nebr. gravel is a possible example of (c). This gravel contains about 90 percent of granite with a thermalexpansion coefficient of $7.2 \times 10^{-6}$ (specimen 67, table 2). About half of the granite, however, is broken into fragments of quartz and orthoclase feldspar, minerals whose thermal-expansion coefficients vary with crystallographic directions between limits of 8.0 and $14.4 \times 10^{-6}$ [14] and of 0.0 and $15.0 \times 10^{-6}[15]$, respectively.

Most of the aggregate materials listed in table 2, excluding single crystals, have thermal expansions close to or within the range of expansions for hardened portland cement. A few, however, have sufficiently different expansivities to warrant some question as to their satisfactory use under severe weather conditions. Where aggregates containing such materials in large amounts are to be used in severe exposures, an examination should be made of the service record in structures having similar exposures. Lacking service background, they should be subjected to laboratory tests of the type described by Pearson [3].

\section{SUMMARY}

Descriptions are given of an apparatus and test procedure used to measure the linear thermal expansion of 137 specimens of rocks and minerals found in concrete aggregates. The mineral constituents and textures of these specimens were determined petrographically. The common aggregate materials, excluding single crystals, were found to have thermal-expansion coefficients ranging from 0.9 to $13.8 \times 10^{-6}$ per degree centigrade within the range of $-20^{\circ}$ to $+60^{\circ} \mathrm{C}$ $\left(-4^{\circ}\right.$ to $\left.+140^{\circ} \mathrm{F}\right)$. The majority of the coefficients are close to the coefficients of hardened portland cement. Crystal orientation, composition differences, and texture are factors which cause variations in the thermal expansions of aggregate materials. Some rocks do not expand uniformly on heating. Under severe weather conditions, the 
expansivity of an aggregate might manifest itself unfavorably in concrete containing one of the following aggregate types: An aggregate having a uniform thermal expansion markedly different from the normal expansion of concrete; an aggregate composed of large crystal fragments having different thermal expansion in different crystallographic directions; an aggregate composed of two or more fractions with widely different expansivities; or an aggregate with irregular expansion.

The authors express their appreciation to the U. S. Bureau of Reclamation office in Denver, the Central Concrete Laboratory of the War Department at Mount Vernon, N. Y., the Tennessee Valley Authority, the State Highway Departments of Washington, California, Missouri, and Nebraska, the Portland Cement Association, and aggregate producers in Tennessee, New York, Ohio, and Louisiana for their cooperation in supplying samples. The authors are indebted to Daniel W. Kessler and Herbert Insley, of this Bureau, for their many helpful suggestions, and to James B. Saunders, also of this Bureau, for his cooperation in developing the testing and recording apparatus.

\section{REFERENCES}

[1] J. C. Pearson, A concrete failure attributed to aggregate of low thermal coefficient, Am. Con. Inst. Proc. 38, 29 (1942).

[2] F. B. Hornibrook, $A$ discussion of "A concrete failure attributed to aggregate of low thermal coefficient," Am. Con. Inst. Proc. 38, 36-3 (1942).

[3] J. C. Pearson, Supplementary data on the effect of concrete aggregate having low thermal coefficient of expansion, J. Am. Con. Inst. 15, 33 (1943).

[4] J. H. Griffith, Thermal expansion of typical American rocks, Iowa Eng. Exp. Sta. Bul. 128 (1936).

[5] T. F. Willis and M. E. De Reus, Thermal volume change and elasticity of aggregates and their effect on concrete, Am. Soc. Testing Materials 39, 919 (1939).

[6] L. H. Koenitzer, Elastic and thermal expansion properties of concrete as affected by similar properties of the aggregate, Am. Soc. Testing Materials 36, pt. II, 393 (1936).

[7] W. H. Souder and P. Hidnert, Thermal expansion of insulating materials, BS Sci. Pap. 15, 387 (1919) S352.

[8] R. E. Davis, A summary of investigations of volume changes in cements, mortars, and concretes produced by causes other than stress, Proc. Am. Soc. Testing Materials 30, pt. I, 668 (1930).

[9] Bureau of Reclamation, Concrete Manual, 27 (Denver, Colo., 1942).

[10] G. E. Merritt, The interference method of measuring thermal expansion, BS J. Research 10, 59 (1933) RP515.

[11] J. B. Saunders, Improved interferometric procedure with application to expansion measurements, J. Research NBS 23, 179 (1939) RP1227.

[12] W. F. Roeser and A. I. Dahl, Reference tablrs for iron-constantan and copperconstantan thermocouples, J. Research NBS 20, 337 (1938) RP1080.

[13] A. Q. Tool, D. B. Lloyd and G. E. Merritt, Dimensional changes caused in glass by heating cycles, J. Am. Ceramic Soc. 13, 641 (1930).

[14] R. B. Sosman, The Properties of Silica, 370 (Chemical Catalog Co., New York, N. Y., 1927).

[15] Kozu and Saiki, Sci. Rep. Toh. Univ. 3, 2, 205 (1925) as quoted in Handbook of Physical Constants, Geol. Soc. Am. Special Papers No. 36, 33 (1942).

[16] J. B. Austin, H, Saini, J. Weigle, and R. H. H. Pierce, Jr., A direct comparison on a crystal of calcite of the $X$-ray and optical interferometer methods of determining linear thermal expansion, Phys. Rev. 57, 931 (1940).

[17] M. A. Swayze, A discussion of " $A$ concrete failure attributed to aggregate of low thermal coefficient." Am. Con. Inst. Proc. 38, 36-12 (1942).

Washington, December 20, 1943. 